1

${ }^{1}$ Department of Entomology and MOA Key Lab of Pest Monitoring and Green Management,
College of Plant Protection, China Agricultural University, Beijing 100193, China.
${ }^{2}$ The Interdisciplinary Ph.D. Program in Genetics, Bioinformatics, and Computational Biology,
Virginia Tech, Blacksburg, VA 24061, USA.
${ }^{3}$ Department of Biochemistry, Virginia Tech, 340 West Campus Drive, Blacksburg, VA 24061 ,
USA

${ }^{1}$ Department of Entomology and MOA Key Lab of Pest Monitoring and Green Management,
College of Plant Protection, China Agricultural University, Beijing 100193, China.
${ }^{2}$ The Interdisciplinary Ph.D. Program in Genetics, Bioinformatics, and Computational Biology,
Virginia Tech, Blacksburg, VA 24061, USA.
${ }^{3}$ Department of Biochemistry, Virginia Tech, 340 West Campus Drive, Blacksburg, VA 24061,
USA

${ }^{1}$ Department of Entomology and MOA Key Lab of Pest Monitoring and Green Management,
College of Plant Protection, China Agricultural University, Beijing 100193, China.
${ }^{2}$ The Interdisciplinary Ph.D. Program in Genetics, Bioinformatics, and Computational Biology,
Virginia Tech, Blacksburg, VA 24061, USA.
${ }^{3}$ Department of Biochemistry, Virginia Tech, 340 West Campus Drive, Blacksburg, VA 24061,
USA

${ }^{1}$ Department of Entomology and MOA Key Lab of Pest Monitoring and Green Management,
College of Plant Protection, China Agricultural University, Beijing 100193, China.
${ }^{2}$ The Interdisciplinary Ph.D. Program in Genetics, Bioinformatics, and Computational Biology,
Virginia Tech, Blacksburg, VA 24061, USA.
${ }^{3}$ Department of Biochemistry, Virginia Tech, 340 West Campus Drive, Blacksburg, VA 24061,
USA

${ }^{1}$ Department of Entomology and MOA Key Lab of Pest Monitoring and Green Management,
College of Plant Protection, China Agricultural University, Beijing 100193, China.
${ }^{2}$ The Interdisciplinary Ph.D. Program in Genetics, Bioinformatics, and Computational Biology,
Virginia Tech, Blacksburg, VA 24061, USA.
${ }^{3}$ Department of Biochemistry, Virginia Tech, 340 West Campus Drive, Blacksburg, VA 24061,
USA

${ }^{1}$ Department of Entomology and MOA Key Lab of Pest Monitoring and Green Management,
College of Plant Protection, China Agricultural University, Beijing 100193, China.
${ }^{2}$ The Interdisciplinary Ph.D. Program in Genetics, Bioinformatics, and Computational Biology,
Virginia Tech, Blacksburg, VA 24061, USA.
${ }^{3}$ Department of Biochemistry, Virginia Tech, 340 West Campus Drive, Blacksburg, VA 24061,
USA

* These authors contributed equally to this manuscript.

\#Corresponding authors. zhujin@vt.edu; zhaozw@cau.edu.cn

\title{
Circadian regulation of microRNA-target chimeras in Drosophila
}

\author{
Xiju Xia ${ }^{{ }^{*}}$, Xiaonan Fu ${ }^{2 *}$, Binbin $\mathrm{Wu}^{1}$, Jinsong Zhu ${ }^{3 \#}$, Zhangwu Zhao ${ }^{1 \#}$
}

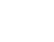

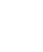

These authors contributed equally to this manuscript. 


\section{Abstract}

MicroRNA is critical coordinator to circadian regulation by silencing gene expression. Although many circadian related miRNAs and some of its target are known, the global functional miRNA-mRNA interaction networks remain poorly understand which is hindered by imperfect base-pairing between miRNA and target mRNA. In this study, we used CLEAR (Covalent Ligation of Endogenous Argonaute-bound RNAs) -CLIP (Cross-Linking and Immuno-Precipitation) to explore the regulatory functions of miRNAs in the circadian system by comparing the miRNA-mRNA interactions between the Drosophila wild-type strain $w^{1118}$ and the Clk mutant $C l k^{j r k}$. We unambiguously identified thousands of miRNA-mRNA interactions from CLEAR-CLIP data set at unprecedented depth in vivo for the first time. Among them, about 300 miRNA-mRNA interactions were involved in the regulation of circadian, in which miRNAs targeting core clock genes $p d p 1$, tim and vri presented distinct changes in response to $C l k^{j r k}$. Particularly, the mir-375-timeless interaction from CLER-CLIP shows important effects on circadian, this functional event occurred in the 1-LNv neurons. Overexpression of mir-375 in tim neurons caused decreases in TIM content resulting in arrhythmicity of daily locomotion and changes of sleep. This present work provides a global view of miRNA targeting in the circadian rhythm.

Keywords: CLEAR-CLIP, miRNAs, circadian rhythm, sleep, $m i R-375$ 


\section{Introduction}

In animals, the intrinsic circadian clock regulates daily rhythms in physiology and behavior, which are entrained by environmental stimuli such as light and temperature ${ }^{1,2}$. This robust timing system maintains rhythmic oscillation even under constant darkness conditions in the fruit fly Drosophila melanogaster.

Sleep is one of the established circadian behavior, which is highly associated with health status ${ }^{3}$. Sleep in Drosophila has been well characterized by certain parameters such as total sleep, sleep bout duration and sleep bout number ${ }^{4-6}$. Throughout the daily sleep-wake cycle, the flies exhibit two peaks of activity - one before lights on and one before lights off. Genetic dissection in Drosophila indicates that sleep is regulated by the circadian clock. The mutations of the core clock genes result in abnormal sleep ${ }^{7,8}$, while the neuropeptide pigment dispersing factor (PDF)-expressing peptidergic clock neurons regulate arousal as well as sleep stability ${ }^{9}$.

The Drosophila rhythmic behavior is maintained by a central clock network in the brain, which is consisted of $\sim 150$ circadian neurons ${ }^{10}$. These clock neurons include ventral lateral neurons (LNvs) (I-LNvs, s-LNvs, and $5^{\text {th }}$ s-LNvs), dorsal lateral neurons (LNds), lateral posterior neurons (LPN) and dorsal neurons (DN1, DN2, and DN3), classified based on anatomical locations and varied expression of core clock genes ${ }^{10-13}$. Among them, the PDF-positive neurons (I-LNvs and s-LNvs) are essential for normal circadian activity and daily sleep. Their ablation results in arrhythmic flies ${ }^{4}$. 
91 (ZT) 14 and a trough at ZT 2 is vital for circadian output ${ }^{10,11}$. Another feedback loop produce the daily rhythm ${ }^{15-17}$.

The highly conserved and widespread microRNAs (miRNAs) play a critical role in post-transcriptional gene regulation ${ }^{18-22}$. They are loaded into Argonaute (AGO) proteins to mediate mRNA cleavage or translational inhibition via base pairing with target mRNAs ${ }^{20,23-25}$. Disruption of the miRNA biogenesis pathway considerably

100 weakens the rhythm in locomotor activity ${ }^{26}$. Although the rhythmically expressed

101 miRNAs have been identified ${ }^{27-29}$, only a limited set of miRNA-target pairs have been

102 verified to affect circadian rhythms ${ }^{26}, 30-33$. Decoding of the miRNA-mRNA

103 interaction network is difficult because of incomplete complementary pairing between

104 miRNAs and targets as well as context-dependent dynamics of the miRNA-target 
105 interactions. As a result, some miRNAs such as miR-124 and miR-959-964 have been

106 shown to be involved in circadian rhythms but without known targets ${ }^{29,34,35}$. Thus, a

107 global identification of circadian-related miRNA-target interactions will greatly

108 improve our understanding of the role of miRNAs in circadian regulation.

109 The effort to accurately predict the miRNA-targets is compromised by the

110 complex and dynamic cellular interaction networks ${ }^{36,37}$. Immunoprecipitation of the

111 miRNA effector protein Argonaute followed by microarray ${ }^{26}$ or RNA-seq analysis ${ }^{38}$

112 narrows down the search scope of potential targets but still relies on bioinformatic

113 analysis to predict miRNA-mRNA pairing. The recently improved experimental

114 method of the CLEAR (Covalent Ligation of Endogenous Argonaute-bound RNAs)

115 -CLIP, which isolates miRNA-mRNA chimeras from endogenous AGO-miRNA

116 -mRNA complexes, permits unambiguous identification of miRNA's targets, which

117 provide a snapshot of true, physiological miRNA-mRNA interactions in $v i v o^{24,39-41}$.

118 To systematically investigate the role of miRNAs in circadian rhythms, we used

119 the CLEAR-CLIP assay in Drosophila. Tens of thousands of miRNA-mRNA

120 interactions were detected highlighting the well-established miRNAs targeting

121 features. Our data indicate that miRNAs extensively fine-tune the expression of

122 circadian-relevant genes. Importantly, we show here that the regulation of tim by

$123 m i R-375$ plays an important role in locomotor activity and sleep. This present work

124 provides a global view of the miRNA-mRNA interactions in circadian rhythms and a

125 solid foundation for future functional studies. 
126

127

129

130

131 post-transcriptional regulation ${ }^{29}$. To uncover the physiological miRNA-mRNA

132 interactions contributing to the circadian rhythm, we applied a modified Ago1

133 CLEAR-CLIP to separately analyze the head and the whole body in $C l k^{j r k}$ and $w^{1118}$

134 flies (Figure S1). The purified Ago1-miRNA-mRNA complexes could be either

135 self-ligated as chimeric reads or remain unchanged mapping to a single locus (CLIP

136 peaks) (Fig. 1A). In total, we obtained 213,404,300 reads from 8 libraries (Table S1A),

137 of which $88.32 \%$ could be mapped to mRNAs, and $1.92 \%$ from miRNA-target

138 chimeras (Fig. 1B). The recovery of previously experimentally supported

139 miRNA-mRNA interactions in Drosophila showed a reliable dataset (Table S1B),

140 exemplified by the verified regulation of hid by bantam ${ }^{43}$ (Fig. 1A).

141 In this study, we discovered 15,825 miRNA-target interactions in total involving

142239 mature miRNAs and 6,540 mRNA transcripts in Drosophila (Table S2A, B).

143 These miRNA- mRNA reads were further classified to miR-first and miR-last

144 chimeras according to the location of miRNA (Table S1C). The miR-first chimeras

$145(92.55 \%)$ were predominant compared with the miR-last chimeras $(7.45 \%)$, and they

146 showed a higher correlation coefficient between miRNA frequency and miRNA 
147 abundance $(r \geq 0.79)$, while the correlation coefficient was low ( $r=\sim 0.45)$ for miR-last

148 chimeras (Fig S2A, B). Thus, we focused exclusively on the miR-first chimeras in

149 subsequent data analysis.

150 The target sequences from the miR-first chimeras were enriched with canonical

151 seed which matched within $50 \mathrm{nt}$ of the ligation sites (Fig. 1C). In addition, the mean

152 of predicted free energy between miRNAs and the matched target mRNAs found in

153 chimeras was lower by $2.8 \mathrm{kcal} \mathrm{mol}^{-1}$ than that in randomly matched pairs (Fig. 1D,

$154 \mathrm{p}<0.001)$. The strong binding energies of chimeric reads suggest that stable chimera

155 stems come from genuine mRNA-miRNA pairing in miRISC rather than from

156 proximity-induced ligation of non-specific RNAs in the solution. These data indicated

157 that the CLEAR-CLIP chimeras reveal a reliable and high-resolution miRNA-target

158 interaction map.

159 The miRNA-target chimeras preserve functional interaction

160 To further evaluate the physiological functions of miRNA-target interactions, we

161 analyzed the binding motif enrichment of let-7-5 $p^{44}, m i R-34-5 p^{45}, m i R-305-5 p^{46}$ and

$162 m i R-276 a^{47}$, which have been investigated in circadian regulation. The enriched 7-mer

163 motifs in the targets of these miRNA were reverse-complementary to the miRNA seed

164 regions, indicating the crucial role of the seed sequences in miRNA targeting (see Fig.

$1652 \mathrm{~A}$ and Fig. S2C). To further examine the evolutionary conservation of

166 chimera-defined target sites, the PhyloP conservation scores of 3'UTR targeting sites

167 were retrieved from multiple alignments of 27 insect genomes. We found that the 
conservation score of chimera-defined targets displayed a marked increase at 6-nt

169 interacted stem relative to flanking regions (Fig. 2B), indicating the close

170 physiological relevance.

171 In addition to assessing the functional outcome of the chimera-identified

172 miRNA-target by CLEAR-CLIP, we also analyzed how the altered RNA levels of

$173 m i R-305$ and $m i R-34$ affected the expression of their target mRNAs based on

174 previously published data related to rhythmicity ${ }^{45}, 46$. In the CLEAR-CLIP dataset, the

175 miR-305 bound to 598 target sites within 555 mRNA transcripts. Overexpression of

176 miR-305 in Drosophila considerably lowers the abundance of mRNAs bearing

177 chimera-defined miR-305 target sites, compared with the mRNAs that had no

178 miR-305 target site $(\mathrm{p}=0.005618)$. And the subset of these mRNAs supported by both

179 chimeras and Ago1 CLIP peaks was also significantly repressed ( $\mathrm{p}=0.0191)$ (Fig.2C).

180 Functional enrichment analysis of $m i R$-305 targets also demonstrated their important

181 roles in sleep (Fig. 2D), which has been observed in the miR-305 mutant $\operatorname{strain}^{46}$.

182 Furthermore, the mRNA levels of the $m i R$-34 targets were significantly elevated in the

183 miR-34 null mutant $(m i R-34-5 p)$, compared with $w^{1118}(\mathrm{p}<0.001)$ (Fig. 2E). In this

184 study, GO enrichment analysis of the chimera-defined miR-34 targets linked $m i R-34$

185 to aging and brain disease (Fig.2F), consistent with the previous report that $m i R-34$ is

186 associated with aging and neurodegeneration ${ }^{45}$. These results verified the

187 physiological significance of the miRNA-mRNA interactions identified by 188 CLEAR-CLIP. 


\section{Clk-oriented miRNA-mRNA interactions are critical for circadian rhythm}

To explore the whole miRNA-mRNA interactions network in the circadian

191 system, we visualized 9325 unique miRNA-target chimeras supported by

192 corresponding Ago1 CLIP peaks with SOM clustering (Fig. S3A). The

193 miRNA-mRNA interactions were significantly changed in $C l k^{\text {jrk }}$ (Fig.3A), implying

194 widespread circadian impact by CLK. Furthermore, by quantitatively identifying the

195 affected miRNA-mRNA interactome (fold change>2), we found that $53.0 \%$ of the

196 circadian relevant regulations were altered, respectively (Fig. 3A). For example, the

197 interaction between miR-276 and tim only appeared in the wildtype strain $w^{1118}$. But

198 the $m i R-34-p d p l$ pairing only happened within the mutant strain $C l k^{j r k}$. These

199 phenomena implicate that the miRNAs involved in circadian regulation are

200 dynamically adapting to the systemic changes.

201 To understand the functional impact of changed miRNA-mRNA interactions, we

202 performed a functional enrichment analysis separately using the significantly

203 up/down-regulated genes. Results showed that many circadian-related functional

204 groups such as sleep and visual perception were top-ranked, especially enriched in the

205 fly-head groups, while chemical synaptic transmission is notably affected after $\mathrm{Clk}$

206 mutation (Fig. S3C).

207 Moreover, by grouping the genes involved in circadian phenotype, we found that

208299 miRNA-mRNA interactions involving 9 core circadian genes were plotted as a

209 miRNA-regulated network (Fig. S3B), in which a majority of interactions appeared to 
210 target the three Clk-downstream genes $p d p 1$, vri and tim (Fig. 3B). To compare the

211 miRNA-involved regulations in these three genes, we retrieved the interaction pattern

212 under different conditions. Results displayed distinct regulatory features in response

213 to $C l k$ disruption (Fig. 3C). The miRNAs targeting $p d p l$ acted in a $C l k$-independent

214 manner. Conversely, the interactions between tim and miRNAs occurred in $w^{1118}$ but

215 were not detectable in $C l k^{j r k}$, with exceptions of $m i R-193-3 p$ and $m i R-2 c-3 p$. Binding

216 of miRNAs to the vri transcript exhibited a mixed pattern of both $C l k$-dependence and

$217 C l k$-independence. The $m i R-276 a / b-3 p, m i R-14-3 p$, and $m i R-210-3 p$ were joint

218 regulators of these three genes (Fig. 3D). Alternatively, $m i R-375-3 p$ and $m i R-305-5 p$

219 only targeted tim in wild type flies (Fig. S4B), in which miR-305-5p had been

220 reported to target tim in several circadian screens ${ }^{48-50}$. These findings suggest that

221 gene regulation by miRNAs has a profound impact on circadian system stability.

\section{Oscillations of $\mathbf{M i r}-375$ expression in pacemaker neurons is affected in $\mathbf{C l k}^{j r k}$}

223 Besides the reads mapped to mRNA, the CLEAR-CLIP dataset also included lots

224 of unligated miRNAs, which has been suggested as a reliable indicator of functional

225 miRNA abundance ${ }^{51}$. To understand the Ago1-bound miRNA changes, we

226 summarized the reads mapped to mature miRNAs. Results showed that only a few

227 miRNAs (miR-375-3p, miR-193-3p, miR-133-3p) related to core circadian genes

228 showed significant changes between $w^{1118}$ and $C l k^{j r k}$. Among them, $m i R-375-3 p$

229 displayed the largest change, with a 3.28-fold increase detected in the fly head (Fig.

230 4A). Thus, we focused on the $m i R-375-3 p$ for further study. 
First, the daily expression of $m i R-375-3 p$ in the fly head was analyzed by

232 qRT-PCR at six time points (ZT0, 4, 8, 12, 16, 20) under LD condition. Results

233 showed that it was rhythmically expressed with the peak at ZT12 in $w^{1118}$, but this

234 pattern disappeared in $C l k^{j r k}$ (Fig. 4B), implying its dependence on $C l k$. To investigate

235 the spatial expression of $m i R-375-3 p$, we utilized a reporter strain

$236($ TI\{GAL4\}mir-375[KO] $\times U A S-M c d 8:: G F P)$ to monitor the expression of mir-375.

237 Results showed that it was expressed in the PDF-expressing I-LNv neurons and 238 co-localized with TIM (Fig.4C).

\section{Mir-375 impacts normal circadian rhythm and sleep}

240 To further evaluate the role of mir-375 in circadian behavior, we overexpressed

241 mir-375 in tim neurons by using iso:tim-gal4 driver. Results showed that the flies with

242 enhanced expression of mir-375 in tim neurons lost daily locomotor rhythm under

243 both LD and DD conditions. Both morning and evening anticipations disappeared

244 after the overexpression of mir-375 (Table 1, Fig.5A-C), while control flies exhibited

245 normal behavioral rhythms (UAS-LUC-mir-375/+: $95.74 \%$ rhythmic, tau=24.5 h,

$246 \mathrm{n}=47$; iso:tim-gal4/+: $100 \%$ rhythmic, tau=24.6 $\mathrm{h}, \mathrm{n}=75$ ). In addition, flies with

247 overexpressed mir-375 showed a decrease in sleep bout duration and an increase in

248 sleep bout number at night compared with the control (Fig.5D, E). These results

249 demonstrate that mir-375 is very important in the regulation of the Drosophila 250 circadian system. 
254 both expressed mir-375 in $1-\mathrm{LNv}$ neurons. In this study, 11 miRNA-tim

255 chimera-defined target sites were founded across the tim transcript (Fig. S4B). Among

256 them, the miR-375 targeting site was located at its CDS region and the chimeras

257 displayed a stable hybridization stem with lower free energy (Fig. 6A).

258 Furthermore, to address whether $m i R-375$ had an effect on the expression of the

259 core clock gene tim, the mRNA levels of tim were detected daily at six time points

$260(\mathrm{ZT} 2,6,10,14,18,22)$ in both flies with mir-375 overexpression (OE) in tim neurons

261 and control flies. Results showed that the amounts of tim mRNA were dramatically

262 decreased in mir-375 OE flies at ZT 10, 14 and 18 (Fig. 6B). Similarly, TIM protein

263 levels in I-LNvs neurons, detected at ZT18 and ZT24 by confocal microscopy, also

264 showed significant decreases (31.1\% at ZT 18 and 54.37\% at ZT24) in mir-375 OE

265 flies compared with control flies (Fig. 6C). This observation was also verified by

266 western blot analysis at ZT18 (Fig. 6D). These results indicate that the TIM in the

267 brain was significantly lower in the mir-375 OE flies than in the control flies.

268 To connect arrhythmic phenotypes caused by overexpressing miR-375 in

269 tim-staining neurons, we simultaneously used the UAS-tim2-5 to rescue this

270 phenotype of overexpressing $m i R-375$, in which the $U A S$-mCD8-GFP served as a

271 control. The results showed that the UAS-tim2-5 could reduce arrhythmicity to $80 \%$ 
272 compared with the $U A S$-mCD8-GFP control, confirming that tim is a real target of

$273 m i R-375$ (Fig. 6E). Taken together, all these data demonstrate that the regulation of

274 tim by mir-375 plays an important role in mediating the daily circadian rhythm.

275

\section{Discussion}

The significance of post-transcriptional regulation in circadian rhythms has been demonstrated by monitoring the core clock gene expression ${ }^{15,52}$ and screening miRNAs involved in rhythmic behavior ${ }^{44,47-49,53-58}$. However, elucidation of the underlying molecular mechanism is impeded because only a few of the predicted miRNA-mRNA interactions have been verified. In this study, we captured miRNA-mRNA interaction pairs directly from fly tissue using the recently developed Ago1 CLEAR-CLIP ${ }^{59}$, 60, which enabled us to detect tens of thousands of miRNA-target interactions. Of these interactions, 75 circadian genes are regulated by 61 miRNAs in the network of 299 interactions (see Fig. 1 and Fig. S3B).

With the first global miRNA-mRNA interaction profiles in Drosophila, we performed multiple lines of evaluation to assess the quality of the whole dataset. First, CLEAR-CLIP recovers many known miRNA-target interactions such as bantam:hid ${ }^{43}$ and $m i R-276 a: t i m^{47}$ (Fig. 1). Second, analysis of interactions recovered from chimeras reveals general bioinformatics features for miRNA targeting, including seed enrichment, stable binding energy and evolutionary conservation (Fig.1, Fig.S3). Third, the chimera-defined target mRNAs exhibit significant changes after manipulating the corresponding miRNA (Fig. 2). Fourth, functional enrichment 
293 analysis of the targets of $m i R-305$ and $m i R-34$ are consistent with the previous

294 phenotypic study of mutant flies ${ }^{45}, 46$. These together substantiate the reliability of the

295 datasets for miRNA-mRNA interaction, which lay the groundwork for further

296 functional studies of miRNAs.

297 In light of chimera-defined miRNA:target pairs, we were able to globally assess

298 the regulatory roles of miRNAs in the whole clock system consisting of input

299 pathways, endogenous clock pacemaker and output pathways (Fig. 3). It is

300 noteworthy that the disruption of $C l k$ has a broad impact on the miRNA-mRNA

301 interaction profile. The shifted pattern points to considerable changes of Ago1 RISC

302 (RNA-induced silencing complex) assemblies in $C l k^{j r k}$. One interpretation is that

303 Ago1-miRNA loading is inefficient in $C l k^{j r k}$ flies because the $C l k$ mutation could

304 abolish some of the cyclic miRNA expression ${ }^{53}$. The miRNA-target interactions may

305 also depend on the availability of miRNA targets, given the central role of $C l k$ in

306 circadian transcription regulation. The drastic changes of circadian mRNAs may alter

307 the Ago loading or retention of the miRNA in RISC ${ }^{61}$. miRNAs regulating PDP1, VRI

308 and TIM present distinct patterns, in which the miRNAs:tim interaction is

$309 C l k$-dependent. Despite the different regulatory connection to $C l k$ expression, the

310 patterns may also result from the combinatorial regulation by multiple miRNAs with

311 diverse cyclic expression patterns. The oscillating circadian mRNAs were regulated

312 by multiple miRNAs to ensure correct timing of expression.

313 Our data clearly indicated that the 9 core circadian genes were regulated by 23 
314 miRNAs in the brain of fruit flies (Fig. S3). Previous miRNA screens have identified

315 a total of 47 miRNAs as relevant to circadian phenotype ${ }^{48-50}, 12$ of those miRNAs

316 were also detected in this study (Fig. S3D). The other miRNAs, which clearly have

317 important roles in circadian rhythm, may indirectly regulate the expression of the

318 circadian genes that have been confirmed to participate in circadian regulation ${ }^{48-50}$. In

319 addition, we linked 10 miRNAs related to circadian rhythm and mapped 9 interaction

320 sites on the tim transcript. These data suggest a complex regulation of miRNAs on

321 rhythmic output.

The current study reports for the first time that $m i R-375$ is involved in circadian

323 rhythm and sleep. At the larval stage of Drosophila, miR-375 is expressed in the

324 salivary glands and hindgut, but detailed expression characterization at adulthood is

325 lacking $^{62}$. In this study, we confirmed its circadian expression in the I-LNv neurons.

326 Overexpressing $m i R-375$ with the tim driver leads to arrhythmic behavior with loss of

327 morning anticipation and normal sleep pattern. Compared with an $81 \%$ arrhythmicity

328 after overexpressing miR-276a in the previous study ${ }^{47}$, we observed a $100 \%$

329 arrhythmicity. In contrast to the peak expression of miR-276a at ZT22, the highest

330 level of miR-375 appeared at ZT12 with significant repression of TIM in the head at

331 ZT18, as detected by immunoblotting (Fig.6). Multiple miRNAs may participate in

332 the spatiotemporal control of tim expression, coordinating the normal rhythmic 333 output.

334 Furthermore, we also found that overexpression of the mir-305 in tim neurons 
335 (iso:tim-gal4×UAS-luc-mir-305) caused a unimodal activity and a phase shift in DD

336 condition, while bimodal activity was maintained in an LD condition (Fig. S5). This

337 phenotype was also observed in $m i R-124$ mutant $^{55,56}$. Our CLEAR-CLIP data suggest

338 that miR-124 targets shaker (sh) and twenty-four (tyf). The chimera-defined

339 miRNA-target interactions imply a broad impact of miRNAs on many aspects of the

340 circadian system and provide a reliable guide to study the function of individual

341 miRNAs. Extensive investigation is required to comprehensively understand the

342 post-transcriptional regulation of circadian rhythms. This study opens a new avenue

343 for a deep understanding of the post-transcriptional regulation of circadian rhythms.

\section{Acknowledgments}

We thank Jeffery Price (University of Missouri at Kansas City) for revision of

347 this manuscript. The work was supported by the Natural Science Foundation of China

348 Grants 31572317 and 31730076 (to ZZ) and National Institutes of Health R01

349 AI122743 (to JZ).

351 Author contributions: J.Z. and Z.Z. designed research; X.X., B.W. and X.F. 352 performed research; X.X., X.F. analyzed data; and X.X., X.F., J.Z. and Z.Z. wrote the 353 paper.

\section{Additional information}

356 Competing financial interests: The authors declare no competing financial interests. 


\section{Materials and Methods}

\section{Fly strains}

Fly strains were maintained on standard molasses-cornmeal-yeast food in a 12L:12D cycle at $25^{\circ} \mathrm{C}$ and $60 \%$ humidity. The fly lines were used in this study as follows: $\quad w^{1118}, \quad C l k^{\text {Irk }}, \quad$ USA-LUC-mir-305/TM3, UAS-LUC-mir-275.mir-305, UAS-LUC-mir-375, UAS-LUC-mir-9c, UAS-Mcd8::GFP, TI\{GAL4\}mir-375[KO], which were purchased from the Bloomington Drosophila Stock Center. The iso:tim-gal4 was obtained from Yirao's lab, UAS-tim2-5 from Jeffrey L. Price's lab and was originally generated by Amita Sehgal's lab..

\section{Ago1 CLEAR-CLIP library construction}

The protocol was adapted from previous reports ${ }^{24,39}$. The collected tissue samples were first ground in liquid nitrogen before ultraviolet irradiation. The purified Ago1-RNA complexes from Drosophila lysates were subjected to stringent wash for removal of nonspecific binding. T4 RNA ligase 1 was added into the complex to promote the formation of miRNA-target chimeras. To track the size of the Ago1-RNA complex, RNA was labeled through ligation with a ${ }^{32} \mathrm{P}$-labeled 3' DNA linker. A subsequent size selection on an SDS polyacrylamide gel was used to isolate the Ago1-miRNA-mRNA complexes (>130 kDa) and recover authentic target RNAs for library construction and sequencing.

\section{Behavior assay and analysis}

Adult male flies (2-5 d old) were used to test locomotor activity rhythms. Flies were entrained under LD for $3 \mathrm{~d}$ and released into constant darkness (DD) for at least $6 \mathrm{~d}$ at $25^{\circ} \mathrm{C}$. Locomotor activity was recorded with Drosophila activity monitors (Trikinetics). Sleep was analyzed by pysolo and GraphPad software. FaasX software was used to analyze behavioral data. Locomotor behavior was analyzed in MATLAB. The details for the experimental protocol and data analysis were described by Chen et $\mathrm{al}^{44}$.

\section{Confocal microscopy}


Adult male flies of 5-10 days were collected and their brains were dissected in 386 phosphate buffered saline (PBS). The brains were subjected to $4 \%$ paraformaldehyde 387 in PBS for $1 \mathrm{~h}$ and then washed three times with the wash buffer $(0.05 \%$ Triton $\mathrm{X}-100$ 388 in PBS) for 10 min at room temperature. Samples were transferred to Blocking buffer 389 (2\% Triton-100, 10\% normal goat serum in PBS) for an overnight incubation at $4{ }^{\circ} \mathrm{C}$ 390 and incubated with primary antibodies (diluted in blocking buffer) overnight at $4^{\circ} \mathrm{C}$. 391 The following primary antibodies were diluted in the blocking buffer: rat-anti-TIM 392 (1:1000, from Jeffrey L. Price), mouse-anti-PDF (1:400, from DSHB). After washing 393 samples three times for 15 min at room temperature, the samples were incubated with 394 secondary antibody $(1: 200)$ at $4^{\circ} \mathrm{C}$ overnight. The samples were imaged on a Leica 395 SP8 confocal microscope. Image J software was used for TIM and PDF quantification.

\section{RNA extraction and qRT-PCR analysis}

397 Fly heads were collected at the indicated time points and stored at $-80^{\circ} \mathrm{C}$ before 398 RNA extraction. Total RNA was extracted from the heads by using Trizol reagent 399 (TIANGEN) following the supplier's instruction. For reverse transcription and 400 real-time PCR of timeless, we used PrimeScript RT reagent kit with gDNA Eraser 401 (TAKARA) and superreal premix plus (SYBR Green) (TIANGEN). The sequences of 402 primers are shown in Table S4. For quantitative analysis of timeless, miR-375 and $2 \mathrm{~s}$ 403 rRNA, we used a miRcute miRNA first-strand cDNA synthesis kit and a miRcute 404 miRNA qRT-PCR detection kit (SYBR Green) (TIANGEN). The miRNA-specific 405 forward primers used for qRT-PCR are also shown in Table S2.

\section{Western blot}

407 Flies' heads were collected at the indicated time points and homogenized in a 1.5 $408 \mathrm{~mL}$ microtube with RIPA lysis buffer (strong) supplemented with proteinase inhibitors. 409 For immunoblot analysis, proteins were transferred to PVDF membrane. After 410 blocking, the membrane was incubated with actin antibody (1:10000) and TIM 411 antibody (1:4000) for $2 \mathrm{~h}$ at room temperature. Image $\mathrm{J}$ was used to calculate band 412 intensity. 


\section{Identification of miRNA-mRNA chimeras}

Ago1 CLEAR-CLIP sequencing reads were preprocessed to remove low-quality reads and 3' adapter sequences using Flexbar ${ }^{63}$. Shorter reads (less than $16 \mathrm{nt}$ ) were discarded. Reads with identical sequences were collapsed. Three random nucleotides

417 were used in the 5' adapter to avoid PCR over-amplification. These random barcodes 418 were trimmed prior to mapping.

419 The clean reads containing miRNA sequences were first defined by mapping mature miRNA sequences against sample libraries using BLAST (v2.3.0) with e-value 421 equals to $0.4^{64}$. Only the best alignment was reported. The remaining sequences excluding miRNAs part were extracted for mapping to the transcripts with BLAST. The reads mapped to rRNA, tRNA and miRNA genes were removed.

\section{Peak calling of CLIP cluster}

The peak calling of CLIP cluster was done as previously described using the pooled reads from three biological samples at each time point ${ }^{65}$. Briefly, overlapped reads were collapsed to form cluster regions. Cubic spline interpolation (Scipy, http://www.scipy.org/) was performed to determine the location and read number of peaks within a cluster ${ }^{66}$. Significant peaks were identified by determining reads number cutoffs with the p-value less than 0.01 using Poisson distribution.

\section{$431 \quad$ Motif analysis}

432 The analysis of overrepresented motifs of Ago1 CLEAR-CLIP by MEME was performed as previously described ${ }^{59}$ with the settings: -dna -mod zoops -max w7 -n motifs 1 . The motifs identified by meme for targets of each miRNA were then aligned to the reverse-complemented miRNA sequence using $\mathrm{FIMO}^{68}$, with the

436 setting-output-p thresh 0.01. High-confidence motifs were generated with FIMO q-value $($ FDR $)<0.05$, and meme Bonferroni-corrected p-value $<0.05$.

438 For de novo motif analysis using Homer, miRNAs with more than 30 unique 439 chimeras-defined sites were kept. The background sequences were randomly selected 440 from other miRNA chimeras. Information score (c) was calculated as previously 
441 described ${ }^{59}$ to retain motifs with $\mathrm{c} \geq 1$. Heatmap with the percentage of the motif 442 presence was created using $\mathrm{R}$ heatmap package.

443 RNA duplex structure prediction and binding free energy

444 The predictions of duplex structure for miRNAs and their target sequences were 445 performed by RNA hybrid. Mutations in chimeras sequences for both miRNA and 446 mRNA were omitted by comparing with the original annotation. The target sequences 447 were extended to $100 \mathrm{nt}$ according to the miRNA seed sites distribution in $D$. 448 melanogaster. The setting of RNA hybrid was modified as previously described to 449 favor the seed pairing ${ }^{59}$. The minimum free energy of each interaction was calculated 450 by RNA hybrid with default settings ${ }^{67}$. The shuffled interactions served as controls for 451 the comparison of free energy distribution. The median free energy for each profile 452 was calculated in $\mathrm{R}$ for comparison.

453 The duplex heatmap was performed with transformed pairing data (base pairing 454 including G-U match as 1, unpaired site as 0). Cluster numbers (k) 3-10 were tested, 455 with $\mathrm{k}=5$ providing the most meaningful set of distinct categories.

456 Analysis of chimera targets in miRNA perturbation experiments

457 The gene expression data after miRNA perturbation was downloaded ${ }^{45,46}$. The $458 \log 2$ fold-change was used to perform cumulative distribution function (CDF) 459 analysis comparing transcripts bearing chimeras-derived miRNA target sites versus 460 those without target sites. The CDFs were also plotted for transcripts in which the 461 chimera-derived miRNA target sites overlap with Ago1 CLIP peaks.

\section{Dynamic analysis of miRNA-mRNA interactions}

463 To quantitatively analyze the temporal profile of miRNA-mRNA interactions, the 464 chimera-supported Ago1 binding peaks were normalized to the total unique reads in 465 each condition ${ }^{69}$. In order to visualize the pattern of difference between each time 466 point, the data were loaded into Gene Expression Dynamics Inspector (GEDIv2.1) 467 with default parameter (26x25 grids) to perform the dynamic analysis ${ }^{70}$. GEDI 468 employs the SOM algorithm to assign the interactions with similar trend into close 
469 tiles. To select the interaction cluster presenting distinct pattern at a certain time point, 470 we first located the tile with maximum value at local spots displaying unique 471 interactions and expanded the spots following gene Density map in GEDI to the edges. 472 Unique interactions for each category were extracted for phenotype enrichment 473 analysis based on FlyBase annotation.

\section{GO and KEGG enrichment analysis}

475 The selected gene list was taken as an input for the functional enrichment analysis 476 with DAVID ${ }^{71}$. The top-ranked GO terms belonging to biological functions were 477 chosen.

\section{Statistics analysis}

Statistics analysis for all indicated data in this study were performed with T-test

480 and $\mathrm{p}$ values considered significant at $\mathrm{p}<0.05$ and extremely significant at $\mathrm{p}<0.001$.

\section{Detailed supplementary information list}

482 Fig. S1 Experimental Procedure of Ago1 CLEAR-CLIP.

483 Fig. S2 miRNAs detected in the Ago-RNA complexes and the miRNA-mRNA 484 chimeras.

485 Fig. S3 The miRNA-mRNA interactions involved in Drosophila circadian system.

486 Fig. S4 The miRNAs involved regulation of tim in Drosophila.

487 Fig.S5 Overexpress mir-305/mir-9c in tim-neuron affects phase of circadian 488 locomotor behavior.

489 Table S1A. Read statistics for 8 drosophila CLEAR-CLIP samples; Table S1B.

490 Experimentally Validated miRNA Targets Found in chimeras supported interactions;

491 Table S1C. miRNA-first and miRNA-last distribution found in CLEAR-CLIP.

492 Table S2A Ago1 CLIP peaks.

493 Table S2B Ago1 CLASH chimeras.

495 References

496 1. Allada, R. \& Chung, B.Y. in Annual Review of Physiology, Vol. 72 605-624 497 (2010). 
2. Johnson, C.H., Elliott, J.A. \& Foster, R. Entrainment of circadian programs. Chronobiology International 20, $741-774$ (2003).

3. Buysse, D.J. Sleep health: can we define it? Does it matter? Sleep 37, 9-17 (2014).

4. Renn, S.C.P., Park, J.H., Rosbash, M., Hall, J.C. \& Taghert, P.H. A pdf neuropeptide gene mutation and ablation of PDF neurons each cause severe abnormalities of behavioral circadian rhythms in Drosophila. Cell 99, 791-802 (1999).

5. Allada, R. \& Chung, B.Y. Circadian organization of behavior and physiology in Drosophila. Annu Rev Physiol 72, 605-624 (2010).

6. Zheng, X. \& Sehgal, A. Probing the relative importance of molecular oscillations in the circadian clock. Genetics 178, 1147-1155 (2008).

7. Rosbash, M., Bradley, S., Kadener, S., Li, Y., Luo, W., Menet, J.S., Nagoshi, E., Palm, K., Schoer, R., Shang, Y. \& Tango, C.H.A. Transcriptional feedback and definition of the circadian pacemaker in Drosophila and animals. Cold Spring Harbor Symposia on Quantitative Biology 72, 75-83 (2007).

8. Allada, R., White, N.E., So, W.V., Hall, J.C. \& Rosbash, M. A mutant Drosophila homolog of mammalian Clock disrupts circadian rhythms and transcription of period and timeless. Cell 93, 791-804 (1998).

9. Rutila, J.E., Suri, V., Le, M., So, W.V., Rosbash, M. \& Hall, J.C. CYCLE is a second bHLH-PAS clock protein essential for circadian rhythmicity and transcription of Drosophila period and timeless. Cell 93, 805-814 (1998).

10. Edery, I., Zwiebel, L.J., Dembinska, M.E. \& Rosbash, M. TEMPORAL PHOSPHORYLATION OF THE DROSOPHILA PERIOD PROTEIN. Proceedings of the National Academy of Sciences of the United States of America 91, 2260-2264 (1994).

11. HunterEnsor, M., Ousley, A. \& Sehgal, A. Regulation of the Drosophila protein timeless suggests a mechanism for resetting the circadian clock by light. Cell 84, 677-685 (1996).

12. Kadener, S., Stoleru, D., McDonald, M., Nawathean, P. \& Rosbash, M. Clockwork Orange is a transcriptional repressor and a new Drosophila circadian pacemaker component. Genes \& Development 21, 1675-1686 (2007).

13. Cyran, S.A., Buchsbaum, A.M., Reddy, K.L., Lin, M.C., Glossop, N.R.J., Hardin, P.E., Young, M.W., Storti, R.V. \& Blau, J. vrille, Pdp1, and dClock form a second feedback loop in the Drosophila circadian clock. Cell 112, 329-341 (2003).

14. Glossop, N.R.J., Houl, J.H., Zheng, H., Ng, F.S., Dudek, S.M. \& Hardin, P.E. VRILLE feeds back to control circadian transcription of Clock in the Drosophila circadian oscillator. Neuron 37, 249-261 (2003).

15. Lim, C. \& Allada, R. Emerging roles for post-transcriptional regulation in circadian clocks. Nat Neurosci 16, 1544-1550 (2013). 
16. Reischl, S. \& Kramer, A. Kinases and phosphatases in the mammalian circadian clock. FEBS Lett 585, 1393-1399 (2011).

17. Bae, K. \& Edery, I. Regulating a circadian clock's period, phase and amplitude by phosphorylation: insights from Drosophila. J Biochem 140, 609-617 (2006).

18. Bushati, N. \& Cohen, S.M. microRNA functions. Annu. Rev. Cell Dev. Biol. 23, 175-205 (2007).

19. He, L. \& Hannon, G.J. MicroRNAs: small RNAs with a big role in gene regulation. Nature reviews. Genetics 5, 631 (2004).

20. Fabian, M.R., Sonenberg, N. \& Filipowicz, W. Regulation of mRNA translation and stability by microRNAs. Annual review of biochemistry 79, 351-379 (2010).

21. Hausser, J. \& Zavolan, M. Identification and consequences of miRNA-target interactions--beyond repression of gene expression. Nature Reviews. Genetics 15, 599 (2014).

22. Chou, C.-H., Chang, N.-W., Shrestha, S., Hsu, S.-D., Lin, Y.-L., Lee, W.-H., Yang, C.-D., Hong, H.-C., Wei, T.-Y. \& Tu, S.-J. miRTarBase 2016: updates to the experimentally validated miRNA-target interactions database. Nucleic acids research 44, D239-D247 (2015).

23. Bartel, D.P. MicroRNAs: target recognition and regulatory functions. cell 136, 215-233 (2009).

24. Moore, M.J., Scheel, T.K., Luna, J.M., Park, C.Y., Fak, J.J., Nishiuchi, E., Rice, C.M. \& Darnell, R.B. miRNA-target chimeras reveal miRNA 3 [prime]-end pairing as a major determinant of Argonaute target specificity. Nature communications 6 (2015).

25. Gorski, S.A., Vogel, J. \& Doudna, J.A. RNA-based recognition and targeting: sowing the seeds of specificity. Nature Reviews Molecular Cell Biology 18, 215-228 (2017).

26. Kadener, S., Menet, J.S., Sugino, K., Horwich, M.D., Weissbein, U., Nawathean, P., Vagin, V.V., Zamore, P.D., Nelson, S.B. \& Rosbash, M. A role for microRNAs in the Drosophila circadian clock. Genes \& development 23, 2179-2191 (2009).

27. Yang, M., Lee, J.-E., Padgett, R.W. \& Edery, I. Circadian regulation of a limited set of conserved microRNAs in Drosophila. BMC genomics 9, 83 (2008).

28. Chen, X. \& Rosbash, M. MicroRNA-92a is a circadian modulator of neuronal excitability in Drosophila. Nature Communications 8 (2017).

29. Vodala, S., Pescatore, S., Rodriguez, J., Buescher, M., Chen, Y.-W., Weng, R., Cohen, S.M. \& Rosbash, M. The oscillating miRNA 959-964 cluster impacts Drosophila feeding time and other circadian outputs. Cell metabolism 16, 601-612 (2012).

30. Chen, W., Liu, Z., Li, T., Zhang, R., Xue, Y., Zhong, Y., Bai, W., Zhou, D. \& 
Zhao, Z. Regulation of Drosophila circadian rhythms by miRNA let-7 is mediated by a regulatory cycle. Nature communications 5, 5549 (2014).

31. Chen, X. \& Rosbash, M. mir-276a strengthens Drosophila circadian rhythms by regulating timeless expression. Proceedings of the National Academy of Sciences 113, E2965-E2972 (2016).

32. Luo, W. \& Sehgal, A. Regulation of circadian behavioral output via a MicroRNA-JAK/STAT circuit. Cell 148, 765-779 (2012).

33. Xue, Y. \& Zhang, Y. Emerging roles for microRNA in the regulation of Drosophila circadian clock. Bmc Neuroscience 19 (2018).

34. Garaulet, D.L., Sun, K., Li, W., Wen, J., Panzarino, A.M., O'Neil, J.L., Hiesinger, P.R., Young, M.W. \& Lai, E.C. miR-124 Regulates Diverse Aspects of Rhythmic Behavior in Drosophila. Journal of Neuroscience 36, 3414-3421 (2016).

35. Zhang, Y., Lamba, P., Guo, P. \& Emery, P. miR-124 regulates the phase of Drosophila circadian locomotor behavior. Journal of Neuroscience 36, 2007-2013 (2016).

36. Schnall-Levin, M., Zhao, Y., Perrimon, N. \& Berger, B. Conserved microRNA targeting in Drosophila is as widespread in coding regions as in 3'UTRs. Proc Natl Acad Sci U S A 107, 15751-15756 (2010).

37. Fan, X. \& Kurgan, L. Comprehensive overview and assessment of computational prediction of microRNA targets in animals. Brief Bioinform 16, 780-794 (2015).

38. Chi, S.W., Zang, J.B., Mele, A. \& Darnell, R.B. Argonaute HITS-CLIP decodes microRNA-mRNA interaction maps. Nature 460, 479-486 (2009).

39. Helwak, A. \& Tollervey, D. Mapping the miRNA interactome by cross-linking ligation and sequencing of hybrids (CLASH). Nature protocols 9, 711-728 (2014).

40. Scheel, T.K., Moore, M.J., Luna, J.M., Nishiuchi, E., Fak, J., Darnell, R.B. \& Rice, C.M. Global mapping of miRNA-target interactions in cattle (Bos taurus). Scientific Reports 7 (2017).

41. Grosswendt, S., Filipchyk, A., Manzano, M., Klironomos, F., Schilling, M., Herzog, M., Gottwein, E. \& Rajewsky, N. Unambiguous identification of miRNA: target site interactions by different types of ligation reactions. Molecular cell 54, 1042-1054 (2014).

42. Renn, S.C., Park, J.H., Rosbash, M., Hall, J.C. \& Taghert, P.H. A pdf neuropeptide gene mutation and ablation of PDF neurons each cause severe abnormalities of behavioral circadian rhythms in Drosophila. Cell 99, 791-802 (1999).

43. Brennecke, J., Hipfner, D.R., Stark, A., Russell, R.B. \& Cohen, S.M. bantam encodes a developmentally regulated microRNA that controls cell proliferation and regulates the proapoptotic gene hid in Drosophila. Cell 113, 25-36 (2003).

44. Chen, W., Liu, Z., Li, T., Zhang, R., Xue, Y., Zhong, Y., Bai, W., Zhou, D. \& 
Zhao, Z. Regulation of Drosophila circadian rhythms by miRNA let-7 is mediated by a regulatory cycle. Nat Commun 5, 5549 (2014).

45. Liu, N., Landreh, M., Cao, K., Abe, M., Hendriks, G.J., Kennerdell, J.R., Zhu, Y., Wang, L.S. \& Bonini, N.M. The microRNA miR-34 modulates ageing and neurodegeneration in Drosophila. Nature 482, 519-523 (2012).

46. Ueda, M., Sato, T., Ohkawa, Y. \& Inoue, Y.H. Identification of miR-305, a microRNA that promotes aging, and its target mRNAs in Drosophila. Genes Cells 23, 80-93 (2018).

47. Chen, X. \& Rosbash, M. mir-276a strengthens Drosophila circadian rhythms by regulating timeless expression. Proc Natl Acad Sci U S A 113, E2965-2972 (2016).

48. You, S., Fulga, T.A., Van Vactor, D. \& Jackson, F.R. Regulation of Circadian Behavior by Astroglial MicroRNAs in Drosophila. Genetics 208, 1195-1207 (2018).

49. Goodwin, P.R., Meng, A., Moore, J., Hobin, M., Fulga, T.A., Van Vactor, D. \& Griffith, L.C. MicroRNAs Regulate Sleep and Sleep Homeostasis in Drosophila. Cell Rep 23, 3776-3786 (2018).

50. Kadener, S., Menet, J.S., Sugino, K., Horwich, M.D., Weissbein, U., Nawathean, P., Vagin, V.V., Zamore, P.D., Nelson, S.B. \& Rosbash, M. A role for microRNAs in the Drosophila circadian clock. Genes Dev 23, 2179-2191 (2009).

51. Flores, O., Kennedy, E.M., Skalsky, R.L. \& Cullen, B.R. Differential RISC association of endogenous human microRNAs predicts their inhibitory potential. Nucleic acids research 42, 4629-4639 (2014).

52. Lerner, I., Bartok, O., Wolfson, V., Menet, J.S., Weissbein, U., Afik, S., Haimovich, D., Gafni, C., Friedman, N., Rosbash, M. \& Kadener, S. Clk post-transcriptional control denoises circadian transcription both temporally and spatially. Nat Commun 6, 7056 (2015).

53. Vodala, S., Pescatore, S., Rodriguez, J., Buescher, M., Chen, Y.W., Weng, R., Cohen, S.M. \& Rosbash, M. The oscillating miRNA 959-964 cluster impacts Drosophila feeding time and other circadian outputs. Cell Metab 16, 601-612 (2012).

54. Cusumano, P., Biscontin, A., Sandrelli, F., Mazzotta, G.M., Tregnago, C., De Pitta, C. \& Costa, R. Modulation of miR-210 alters phasing of circadian locomotor activity and impairs projections of PDF clock neurons in Drosophila melanogaster. PLoS Genet 14, e1007500 (2018).

55. Zhang, Y., Lamba, P., Guo, P. \& Emery, P. miR-124 Regulates the Phase of Drosophila Circadian Locomotor Behavior. J Neurosci 36, 2007-2013 (2016).

56. Garaulet, D.L., Sun, K., Li, W., Wen, J., Panzarino, A.M., O'Neil, J.L., Hiesinger, P.R., Young, M.W. \& Lai, E.C. miR-124 Regulates Diverse Aspects of Rhythmic Behavior in Drosophila. J Neurosci 36, 3414-3421 (2016).

57. Chen, X. \& Rosbash, M. MicroRNA-92a is a circadian modulator of neuronal 
excitability in Drosophila. Nat Commun 8, 14707 (2017).

58. Xue, Y. \& Zhang, Y. Emerging roles for microRNA in the regulation of Drosophila circadian clock. BMC Neurosci 19, 1 (2018).

59. Moore, M.J., Scheel, T.K., Luna, J.M., Park, C.Y., Fak, J.J., Nishiuchi, E., Rice, C.M. \& Darnell, R.B. miRNA-target chimeras reveal miRNA 3'-end pairing as a major determinant of Argonaute target specificity. Nat Commun 6, 8864 (2015).

60. Helwak, A. \& Tollervey, D. Mapping the miRNA interactome by cross-linking ligation and sequencing of hybrids (CLASH). Nat Protoc 9, 711-728 (2014).

61. Flores, O., Kennedy, E.M., Skalsky, R.L. \& Cullen, B.R. Differential RISC association of endogenous human microRNAs predicts their inhibitory potential. Nucleic Acids Res 42, 4629-4639 (2014).

62. Poy, M.N., Hausser, J., Trajkovski, M., Braun, M., Collins, S., Rorsman, P., Zavolan, M. \& Stoffel, M. miR-375 maintains normal pancreatic alpha- and beta-cell mass. Proc Natl Acad Sci U S A 106, 5813-5818 (2009).

63. Dodt, M., Roehr, J.T., Ahmed, R. \& Dieterich, C. FLEXBAR-Flexible Barcode and Adapter Processing for Next-Generation Sequencing Platforms. Biology (Basel) 1, 895-905 (2012).

64. Altschul, S.F., Gish, W., Miller, W., Myers, E.W. \& Lipman, D.J. Basic local alignment search tool. J Mol Biol 215, 403-410 (1990).

65. Zisoulis, D.G., Lovci, M.T., Wilbert, M.L., Hutt, K.R., Liang, T.Y., Pasquinelli, A.E. \& Yeo, G.W. Comprehensive discovery of endogenous Argonaute binding sites in Caenorhabditis elegans. Nat Struct Mol Biol 17, 173-179 (2010).

66. Haecker, I., Gay, L.A., Yang, Y., Hu, J., Morse, A.M., McIntyre, L.M. \& Renne, R. Ago HITS-CLIP expands understanding of Kaposi's sarcoma-associated herpesvirus miRNA function in primary effusion lymphomas. PLoS Pathog 8, e1002884 (2012).

67. Kruger, J. \& Rehmsmeier, M. RNAhybrid: microRNA target prediction easy, fast and flexible. Nucleic Acids Res 34, W451-454 (2006).

68. Grant, C.E., Bailey, T.L. \& Noble, W.S. FIMO: scanning for occurrences of a given motif. Bioinformatics 27, 1017-1018 (2011).

69. Hafner, A., Stewart-Ornstein, J., Purvis, J.E., Forrester, W.C., Bulyk, M.L. \& Lahav, G. p53 pulses lead to distinct patterns of gene expression albeit similar DNA-binding dynamics. Nat Struct Mol Biol 24, 840-847 (2017).

70. Eichler, G.S., Huang, S. \& Ingber, D.E. Gene Expression Dynamics Inspector (GEDI): for integrative analysis of expression profiles. Bioinformatics 19, 2321-2322 (2003).

71. Huang da, W., Sherman, B.T. \& Lempicki, R.A. Systematic and integrative analysis of large gene lists using DAVID bioinformatics resources. Nat Protoc 4, 44-57 (2009). 

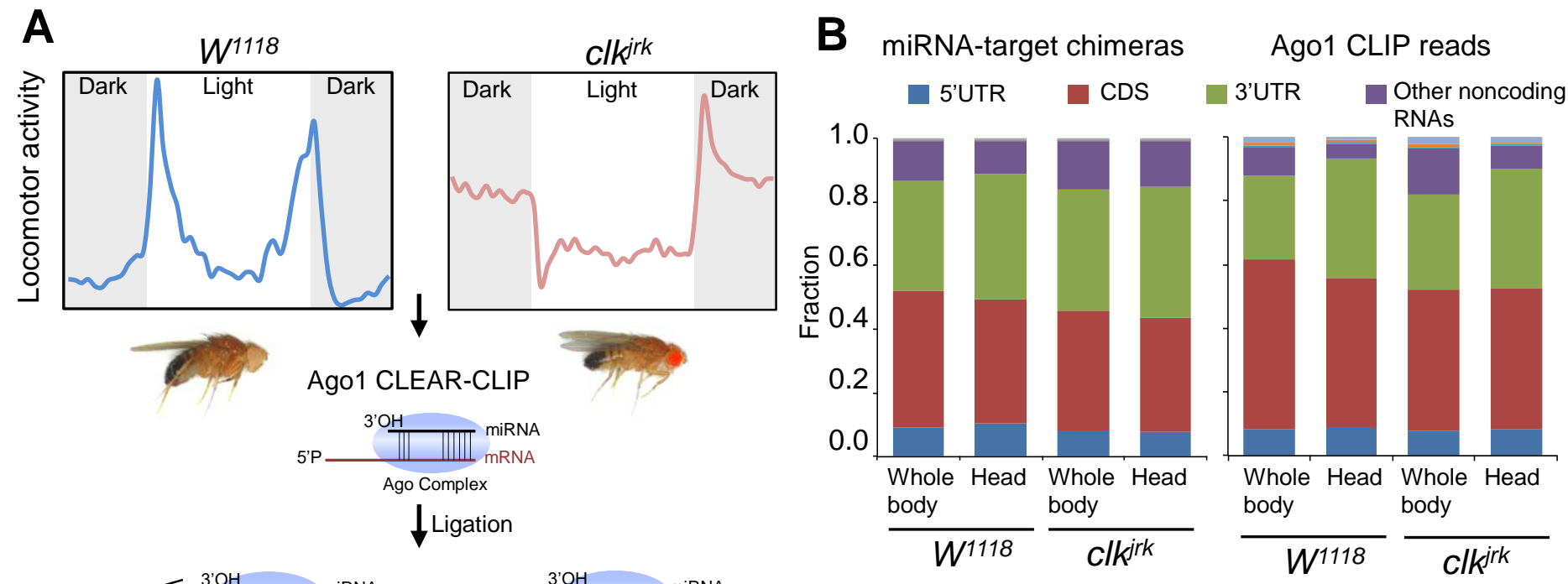
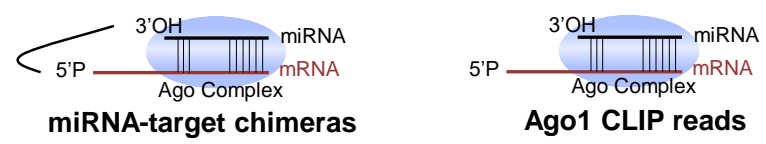

chimeral: TGAGATCATTTTGAAAGCTGATTATTGCTAATTAGTTTTCACAATGATCTCGGTAAAGTTTTGTGGCC chimera2: TGAGATCATTTTGAAAGCTGATA----------GTTTTCACAATGATCTCGGTAAAGTTTTGTGGCC dme-bantam hid-3' UTR

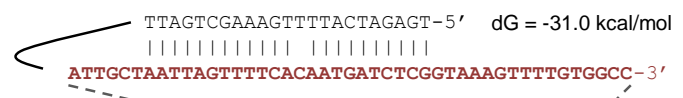

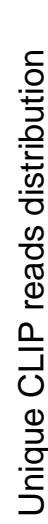
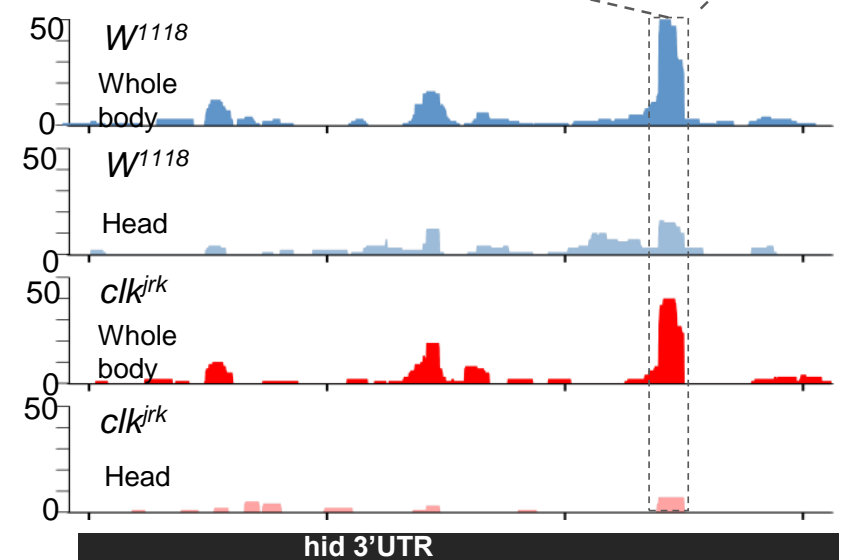

C
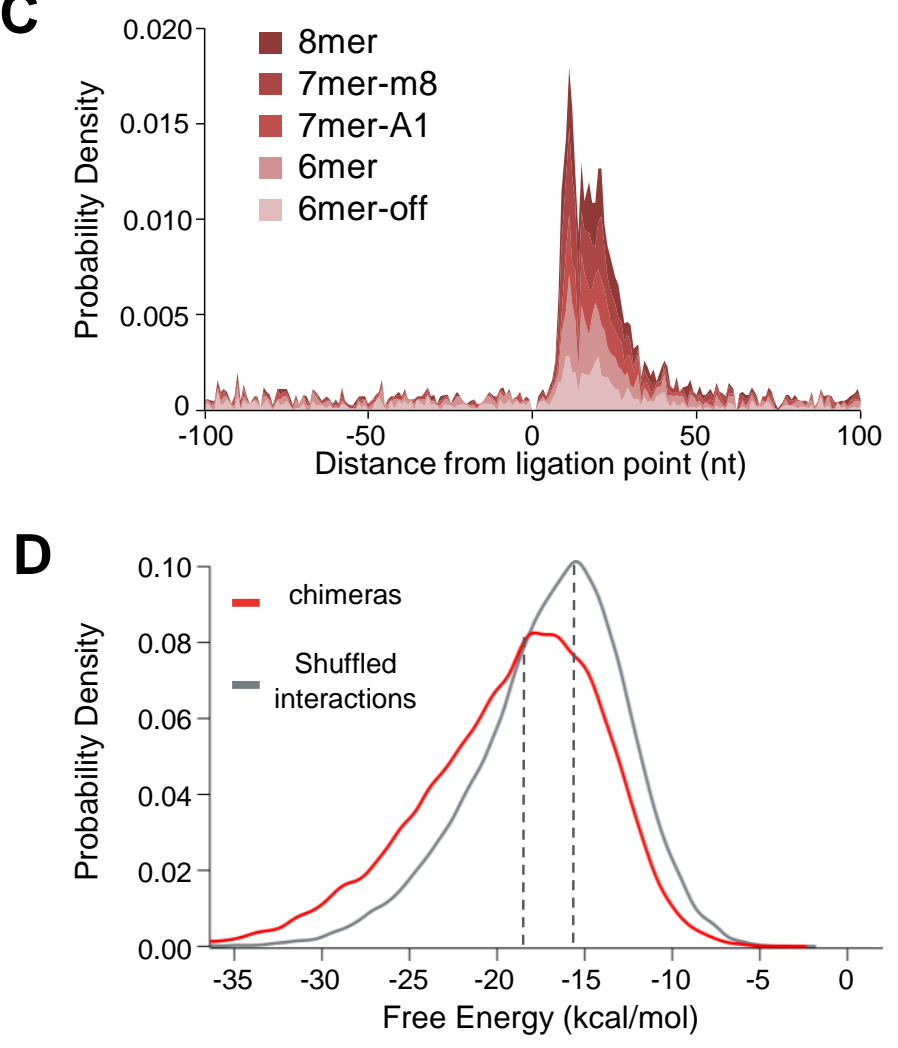

Fig. 1 Ago1 CLEAR-CLIP decodes miRNA-mRNA interactions in Drosophila.

(A) Schematics of CLEAR-CLIP to detect miRNA-mRNA interactions in Drosophila. Wild-type $W^{1118}$ and Clk mutant clkirk fiies with arrhythmic phenotype were collected. The UV-crosslinked sample was homogenized in lysis buffer. Ago1-RNA complex was immunoprecipitated and incubated with T4 RNA ligase to favor the direct ligation of miRNA with its target mRNA fragments. The recovered RNAs were subjected to library construction and sequencing. With the bioinformatics analysis, the chimeric reads could be identified along with the Ago1 binding reads, exemplified by the known bantam targeting hid.

(B) The fraction of different RNA classes annotated to Drosophila for miRNA-target chimeras and Ago CLIP reads.

(C) Density plot of canonical miRNA seed matches in target regions relative to ligation site for all the chimeras.

(D) The median predicted binding energy between miRNA and matching target mRNA found in chimeras was stronger by over $2.8 \mathrm{kcal} \mathrm{mol}-1$ than in randomly matched pairs. 
A

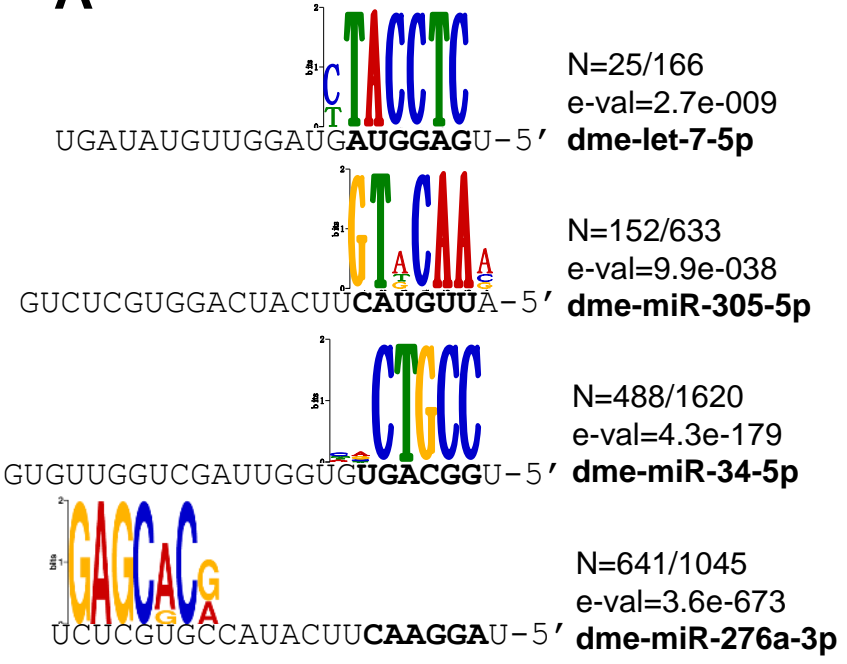

B

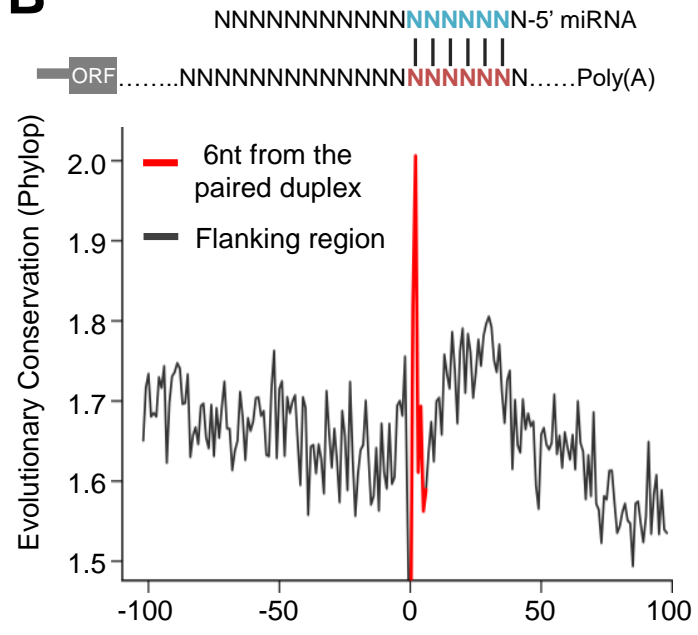

Distance from 5'end of stem in encoded mRNA (nt)
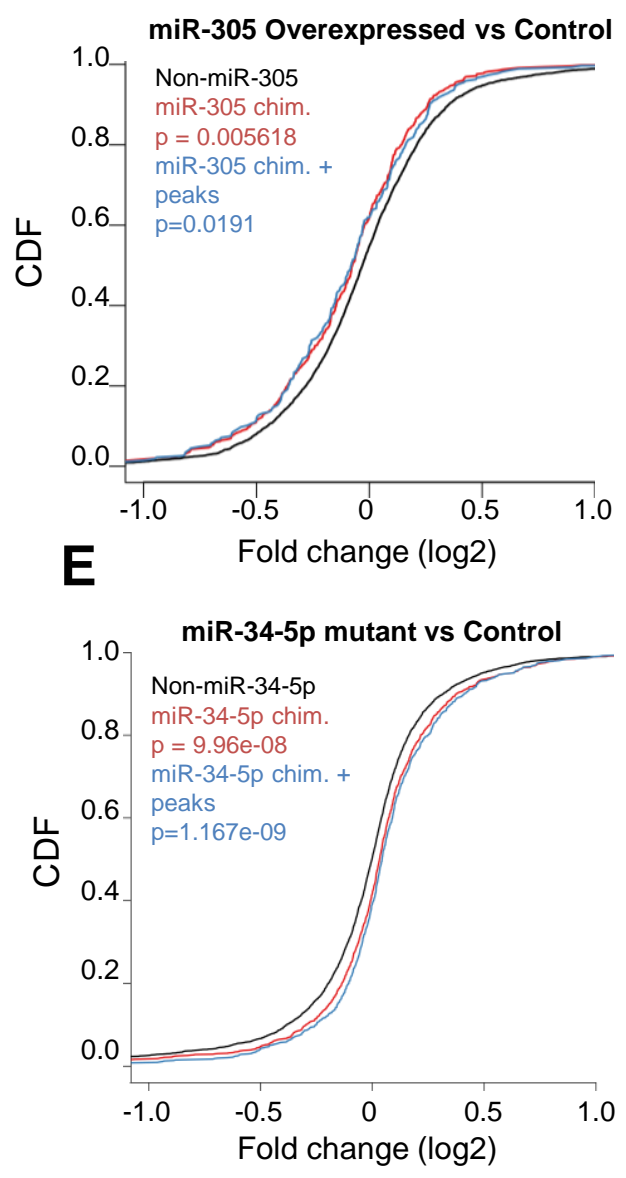

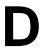

D

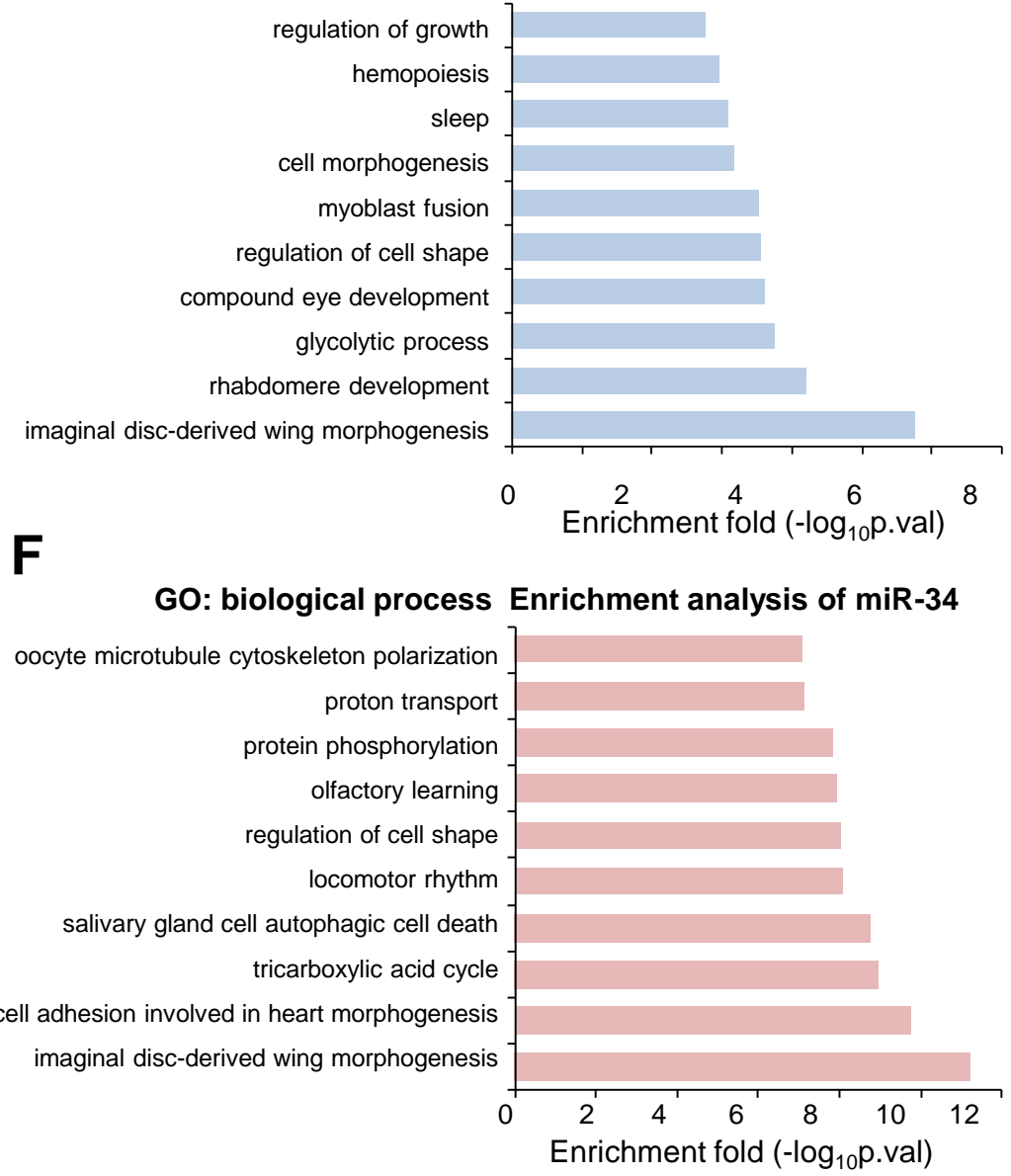

Fig.2 miRNA-target chimeras preserve physiological relevant interactions

(A)Enriched miRNA binding motifs by chimeras-defined target sites. $n$, number of motifs found/total number of targets analyzed. E-val, e-value of the motif returned by MEME. Most motifs are complementary to the miRNA seed (bold).

(B) Average conservation score of perfect seed matches from miRNA:target interactions located in 3'UTR defined by chimeras. The Phylop conservation score for 27 insect species was downloaded from UCSC.

(C) Cumulative distribution function (CDF) plot shows $\log _{2}$-transformed expression differences for mRNAs identified in miR-305 chimeras comparing the other mRNAs which is not defined as miR-305 targets. The analysis was performed using the transcriptome measurement in miR-305 overexpressed fly versus the wild type control. $P$-values was calculated from Kolmogorov-Smirnov testing.

(E) Cumulative distribution function (CDF) plot shows the change in transcript level after knocking out miR-34-5p. The similar analysis as (A) presents the mRNA expression distribution detected in miR-34-5p chimeras, miR-34-5p chimeras supported by Ago1 peaks and the other mRNAs.

$(\mathrm{D}, \mathrm{F}) \mathrm{GO}$ enrichment analysis of chimeras-defined miRNA targets. The top ten biological process terms were plotted. 

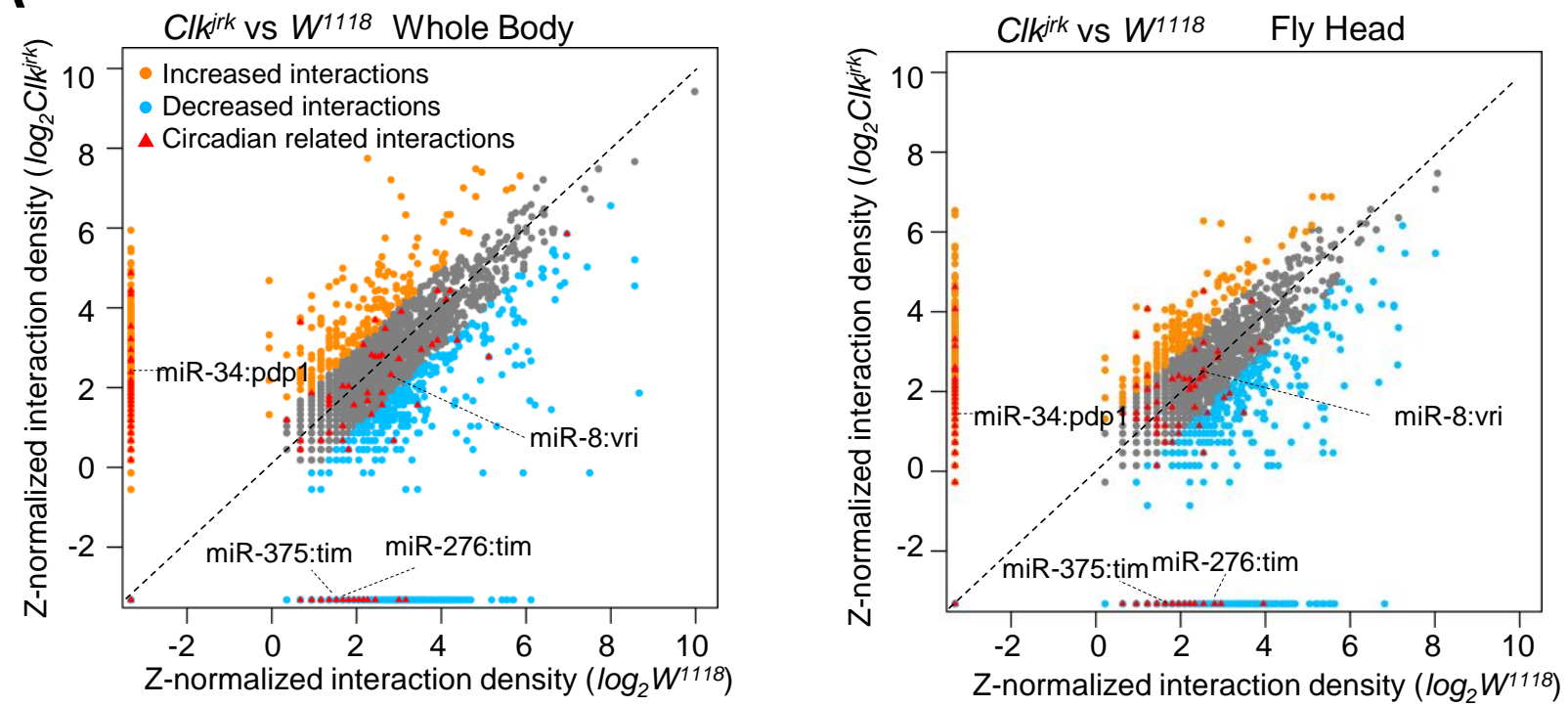

B

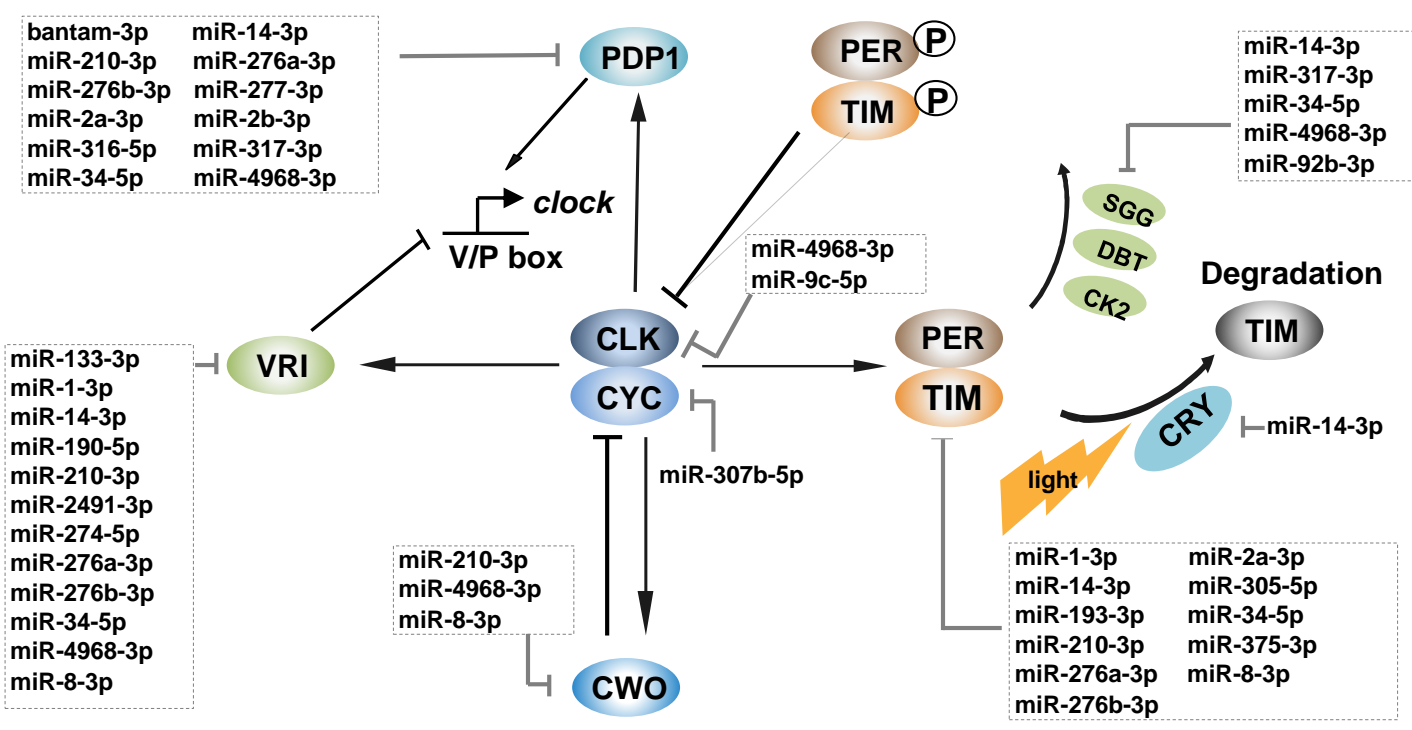

C
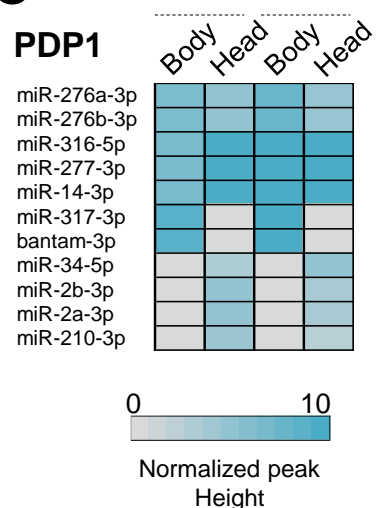
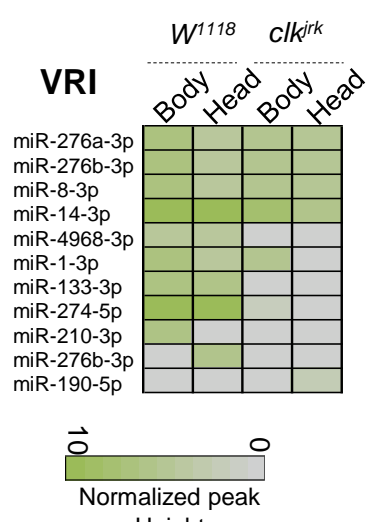

Height
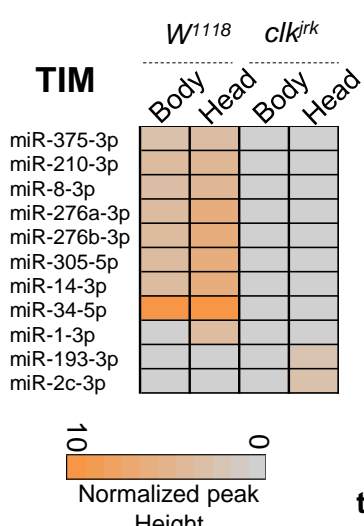

Height
D

miRNAs targeting PDP1

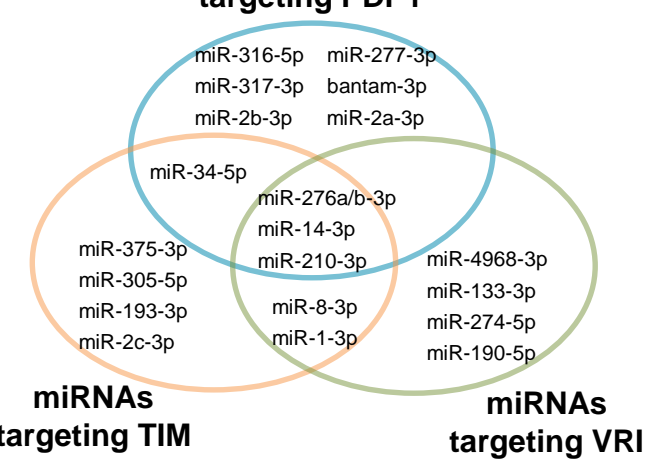

Fig. 3 miRNA-mRNA interactions regulates circadian rhythm in Drosophila

(A) Scatter plot shows the change of miRNA-mRNA interaction comparing Clkirk with $W^{1118}$. The interactions with 2 fold changes are considered as significance.

(B) The miRNAs participated regulation in the core network of circadian system. All the miRNA-mRNA interactions were supported by Ago1 peaks.

(C) The heatmap shows the change of miRNA-mRNA interactions for PDP1, TIM and VRI at each condition.

(D) Venn diagram shows the subsets of miRNAs contribute to the regulation of three core circadian genes. 
A Ago1-bound miRNAs

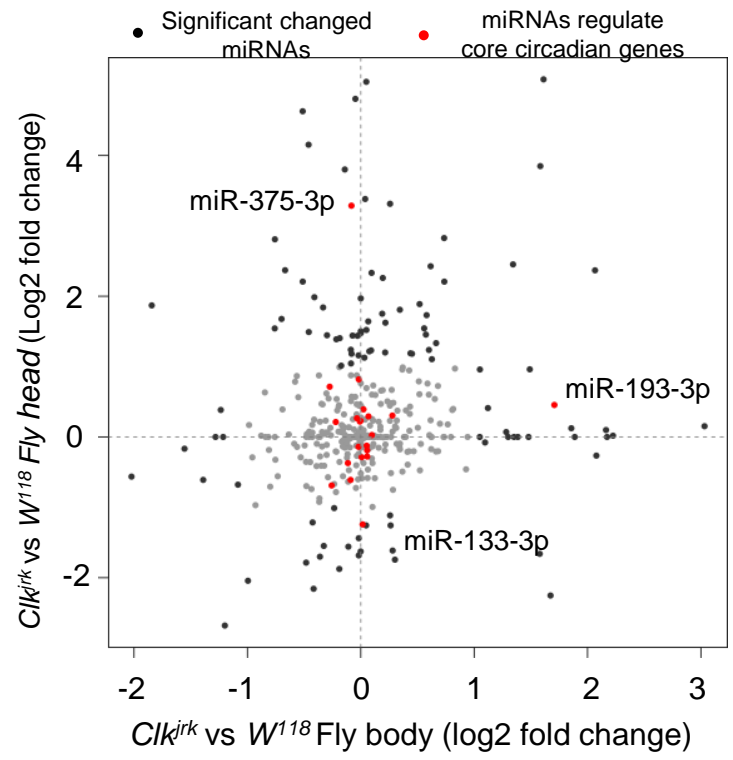

B

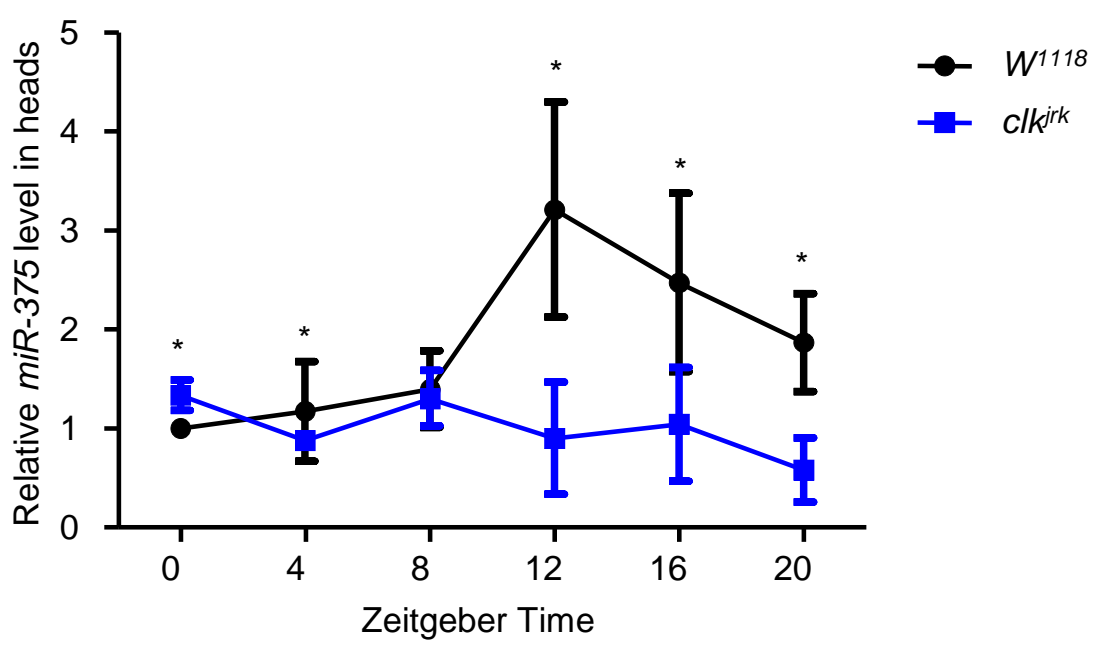

TI\{Gal4]mir-375KO::mGFP

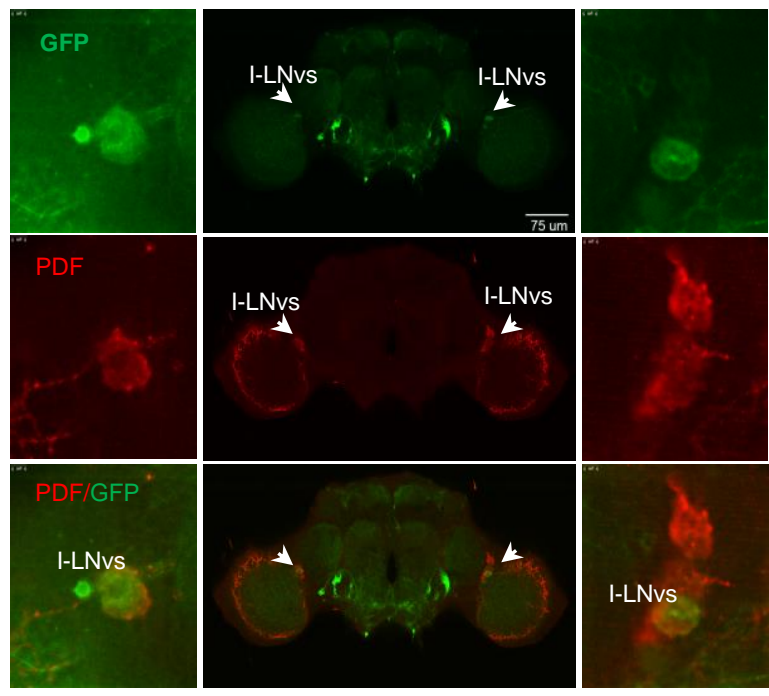

Fig. 4, mir-375 oscillations in pacemaker neurons.

(A) The changes of Ago1-bound miRNAs abundance between C/k $k^{\text {rk }}$ and $W^{1118}$ comparing whole fly and head.

(B) The levels of miR-375 are altered in C/kirk mutant flies' heads.

(C) $\mathrm{w}^{*}$; TI\{GAL4\}mir-375KO expression in Drosophila brain. w $^{*}$; TI\{GAL4\}mir-375KO flies were crossed with UAS-mGFP files. 
A

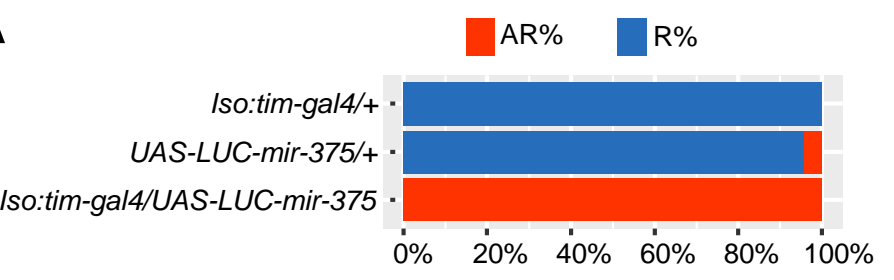

B

Iso:tim-gal4/+ $\mathrm{Tau}=24.6 \pm 0.09$

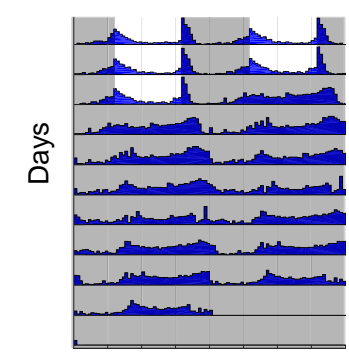

C

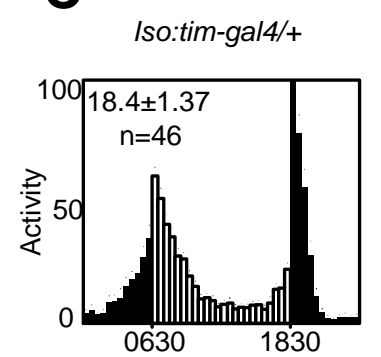

UAS-LUC-mir-375/+ $\mathrm{Tau}=24.5 \pm 0.08$
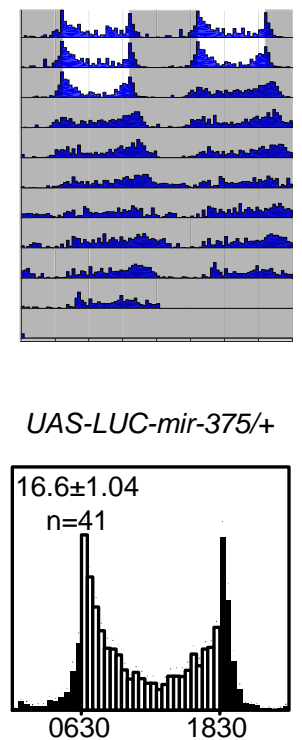

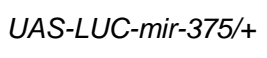

Iso:tim-gal4/UAS-
LUC-mir-375

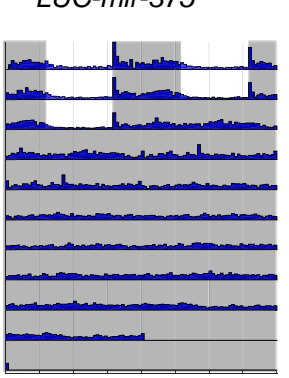

E Total sleep

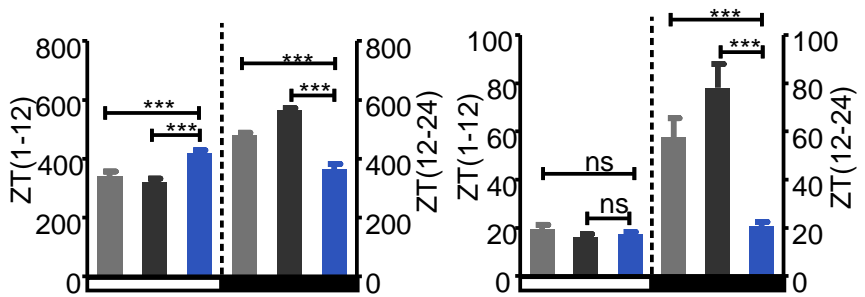

Iso:tim-gal4/UAS-

LUC-mir-375

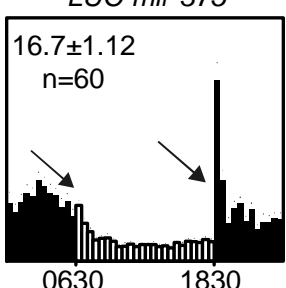

D

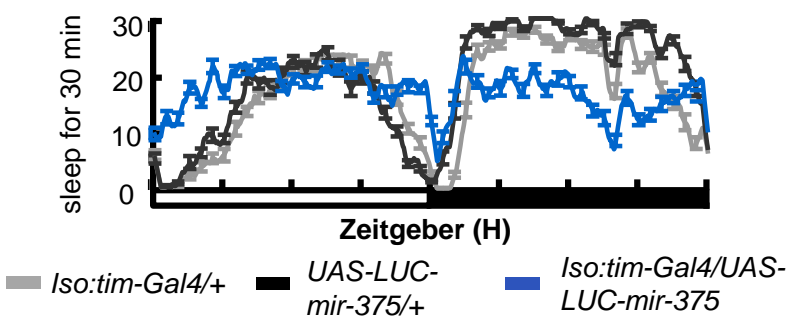

LUC-mir-375 

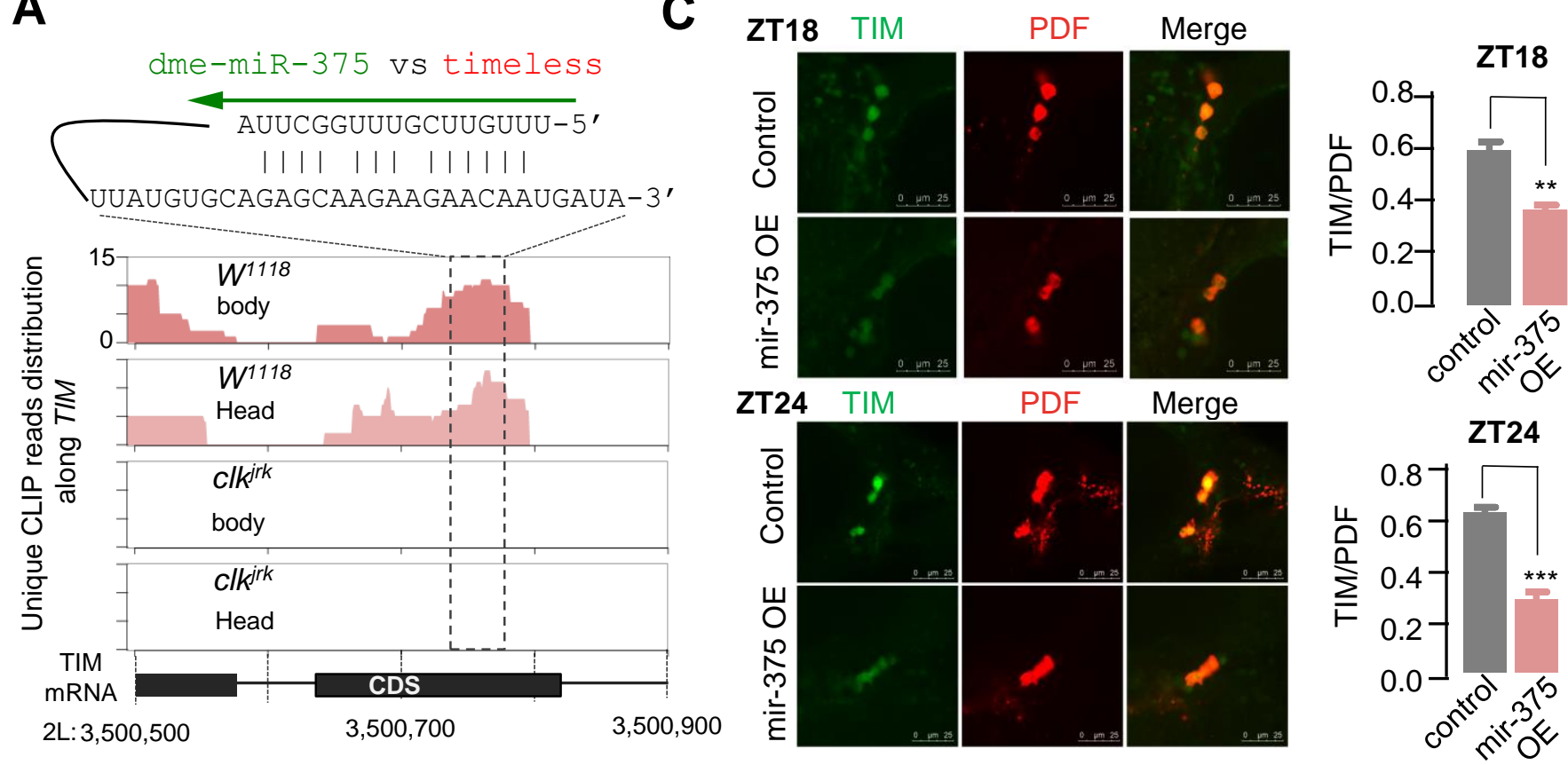

B
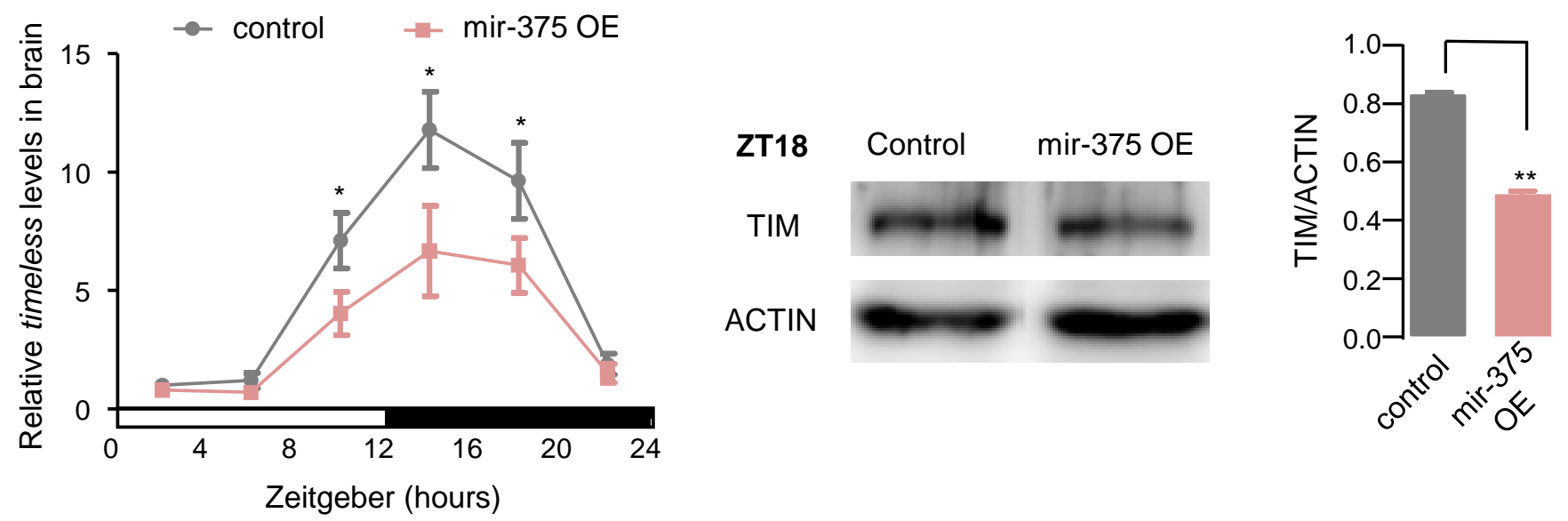

E

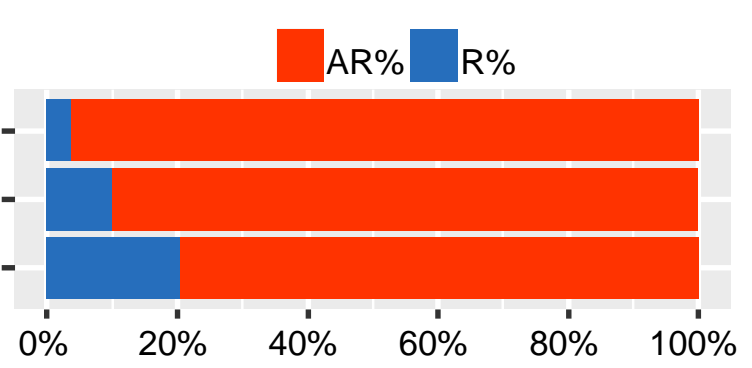

Fig.6 Tim is a target of mir-375.

(A) The Clk-dependent interaction of miR-375 targeting timeless in its coding region. The duplex defined in chimeras was predicted by RNAhybrid.

(B) Levels of timeless mRNA are altered in miR-375 OE flies. The relative expression levels were normalized to actin levels and were further normalized to control at ZT2. (error bar indicate SEM, ${ }^{*} P<0.05$; two-tailed t test. )

(C) Immunostaining of control (iso-tim-gal4/+; UAS-LUC-mir-375/+) and mir-375 OE (iso-tim-gal4/UAS-LUC-mir-375) flies against TIM (green) and PDF (red) at ZT18 (upper) and ZT24 (lower). Tim signal is normalized to the PDF signal. ( $n=10-17$ hemisphere; error bar indicate SEM, ${ }^{* \star} P<0.01$; ${ }^{* \star \star \star} P<0.0001$; two-tailed $t$ test. )

(D) Western blot against TIM in control and mir-375 OE flies ZT 17, ACTIN is used as loading control, TIM levels are normalize to ACTIN levels. Protein signals are quantitated by using image J. ( $n=4$, error bar indicate $S E M$, ${ }^{* *} P<0.01$; two-tailed t test. )

(E) Overexpression of timeless levels recues arrhythmic behavior in mir-375OE flies. AR\% represents the percentage of arrhythmic flies, R\% represents the percentage of rhythmic flies. 
A
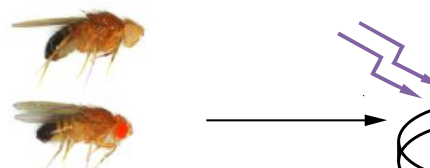

UV $254 \mathrm{~nm}$ irradiation
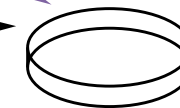

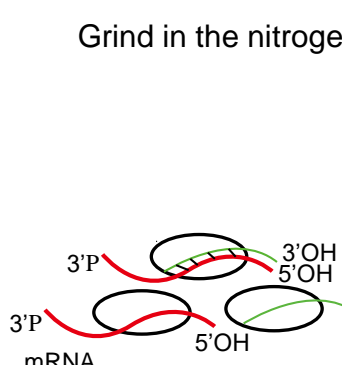

mRNA

T4 PNK treatment
1. tissue lysis, DNase

2. AGO1 IP

3. High stringency washes

4. RNase treatment

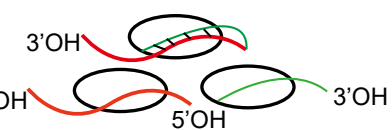

Self ligation

1. Dephosphrylation (CIP)

2. 3'linker ligation
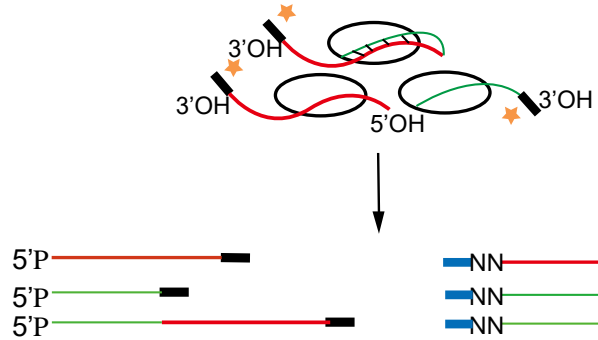

RNA extraction and purification
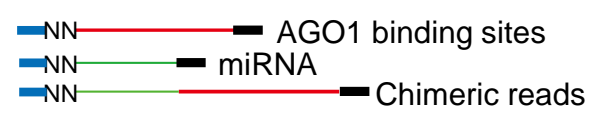

5 'linker ligation

1. Reverse transcription

2. Library construction

Sequencing
B
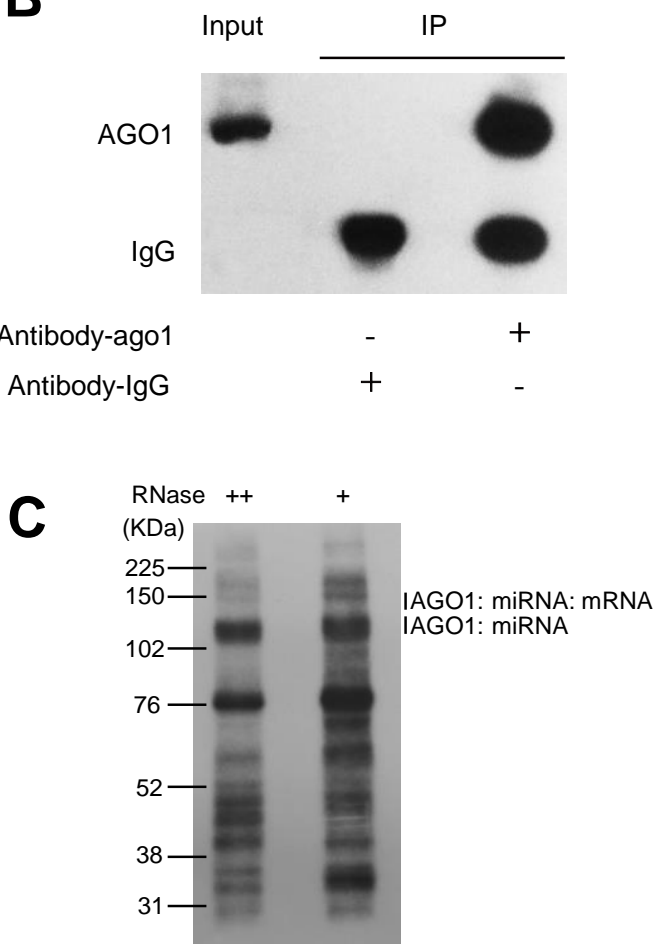

D

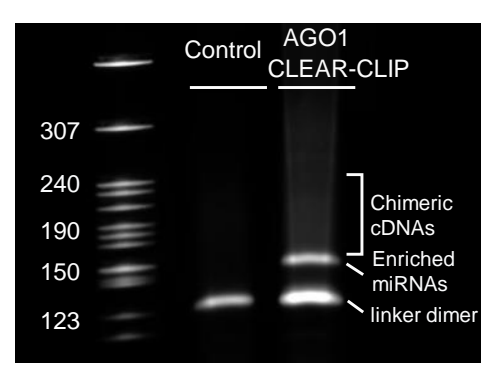

\section{Fig. S1 Experimental Procedure of Ago1 CLEAR-CLIP.}

(A) In Ago1 CLEAR-CLIP, the tissue lysates were prepared from UV cross-linked flies. Endogenous Ago1 was immunopurified and stringently washed to remove non-specific interactions. The RNA ends in the Ago1-complexes were treated with T4 Polynucleotide Kinase and ligated together. RNAs associated with Ago1 were then further purified by size selection using the SDS-PAGE gel and recovered for library construction and sequencing (material and method).

(B) Western blot detect Ago1 in input sample, IP with antibody-Ago1 and antibody-IgG.

(C) Autoradiogram of the Ago1 associated RNA after digestion with different RNase.

(D) PCR products amplified using the recovered RNAs after linker ligation. 

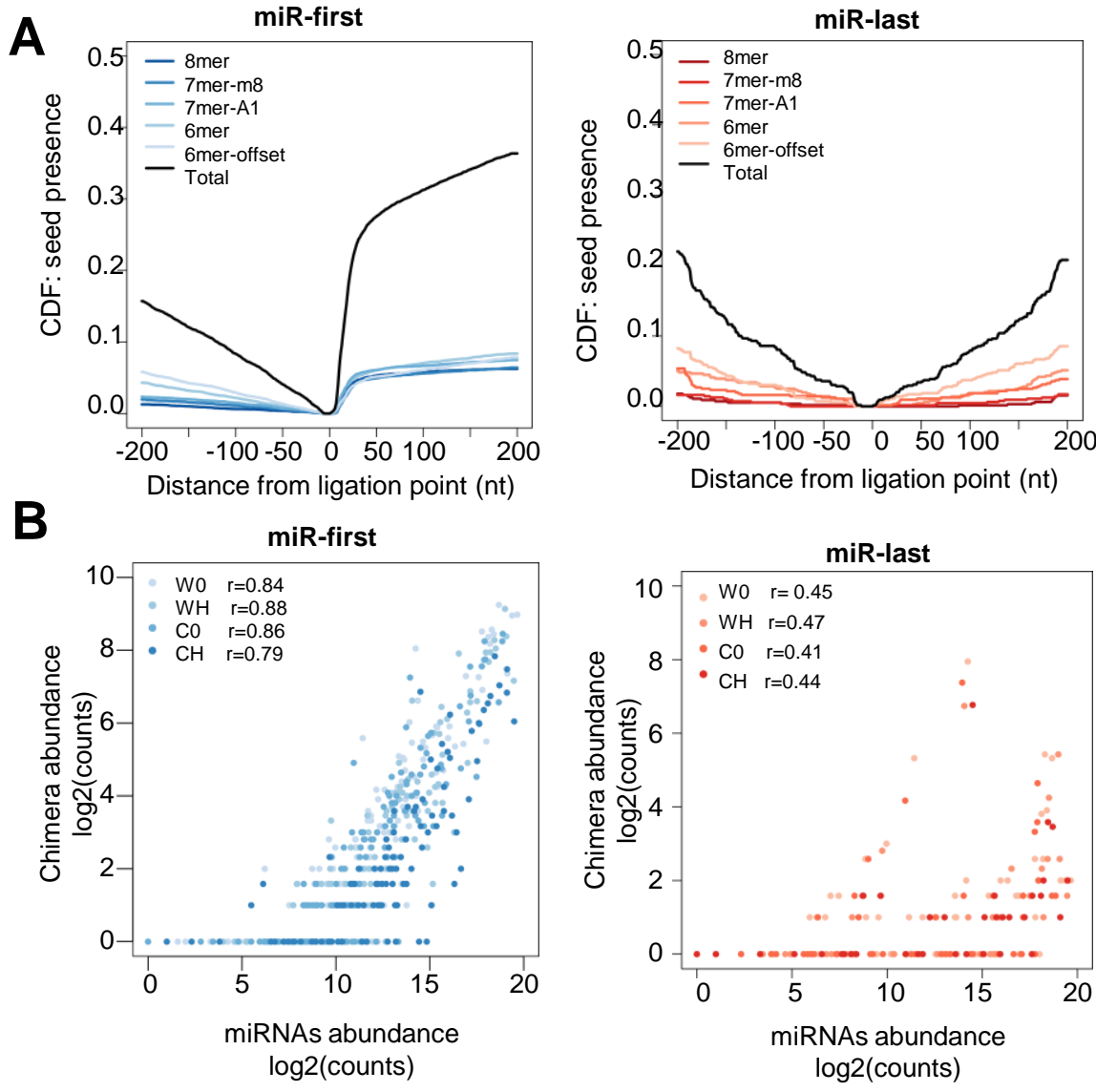

C

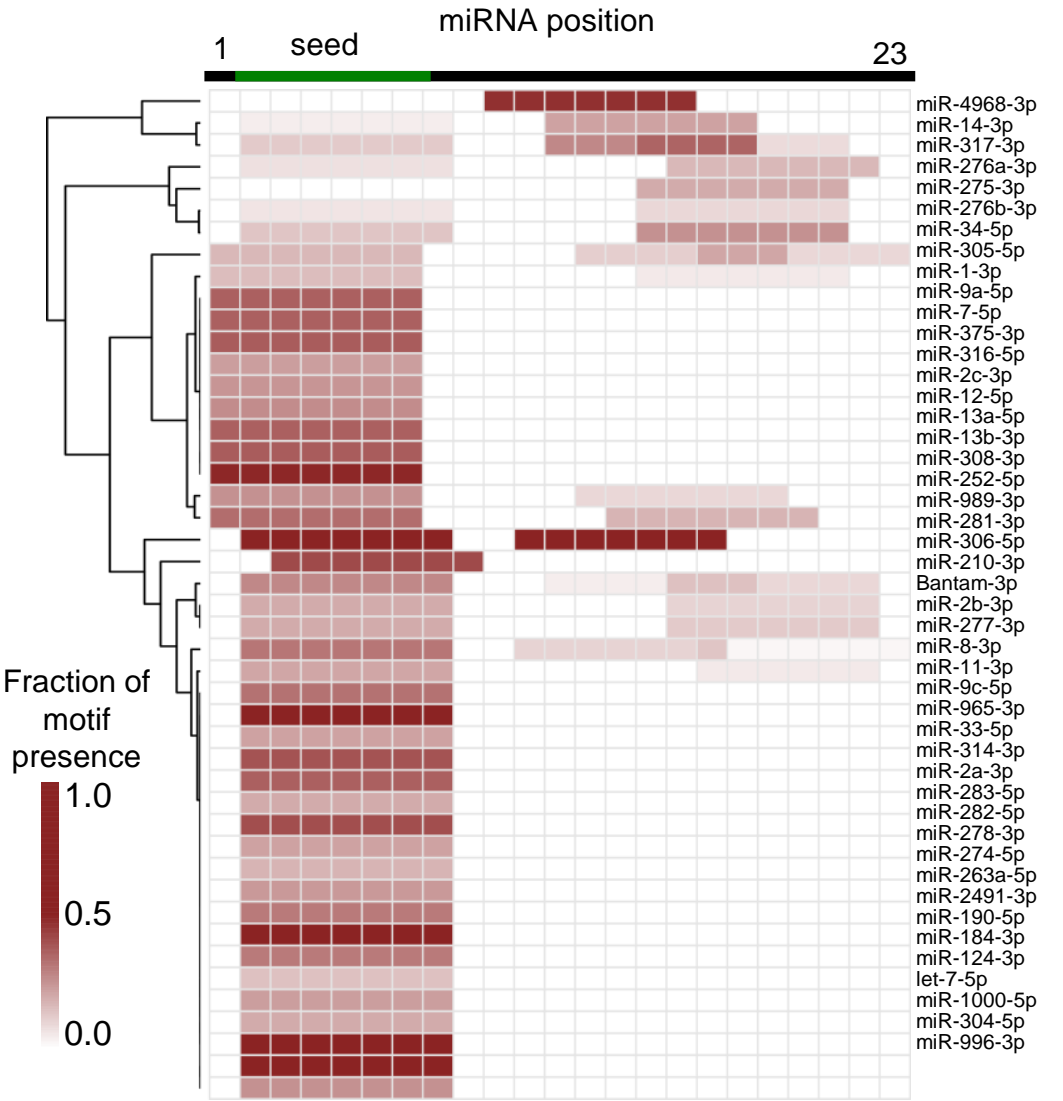

Fig. S2 miRNAs detected in the Ago-RNA complexes and the miRNA-mRNA chimeras.

(A) The cumulative distribution function of canonical miRNA seed matches in target regions relative to ligation site for miR-first and miR-last chimeras.

(B) Correlation plots of miRNA abundance from the whole CLEAR-CLIP libraries and their chimeras. Pearson's correlation coefficients are shown for each condition.

(C) The heatmap shows the distribution of significant enriched 7 mer motifs through de novo analyzing all chimeras detected for individual miRNA. The colour intensity is proportional to abundance of 7 mer motif in target sequences. The hierarchical clustering was performed in $\mathrm{R}$ package gplots2. 
A

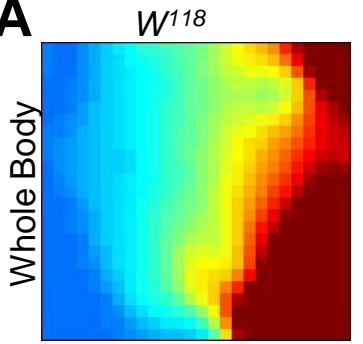

clkjrk
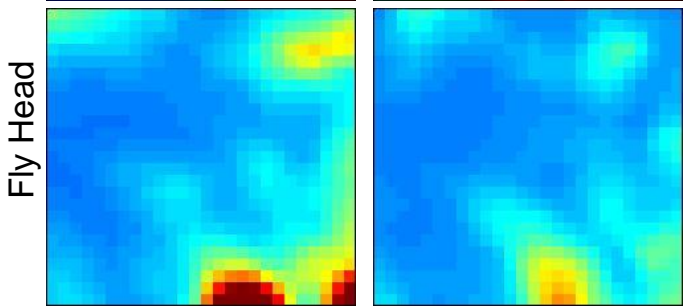

C GO: biological process wing morphogenesis sarcomere organization visual perception regulation of cell shape

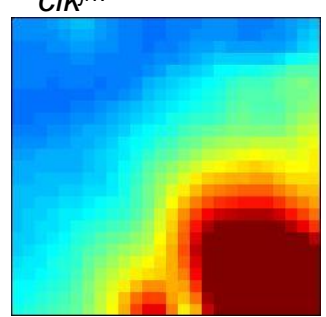

cell adhesion myofibril assembly long-term memory glucose metabolic process septate junction assembly phototransduction phototransduction
cell morphogenesis

cell morphogenesis
cytoplasmic translation locomotor rhythm chemical synaptic transmission dorsal closure

regulation of cell shape myofibril assembly wing morphogenesis cell morphogenesis actin cytoskeleton organization muscle attachment cell autophagic cell death cytoplasmic translation visual perception

protein phosphorylation
pctin filament organization

nervous system development

phagocytosis glucose metabolic process muscle cell cellular homeostasis axon guidance epithelial cell apical/basal polarity

Whole Body

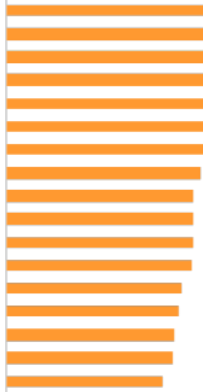

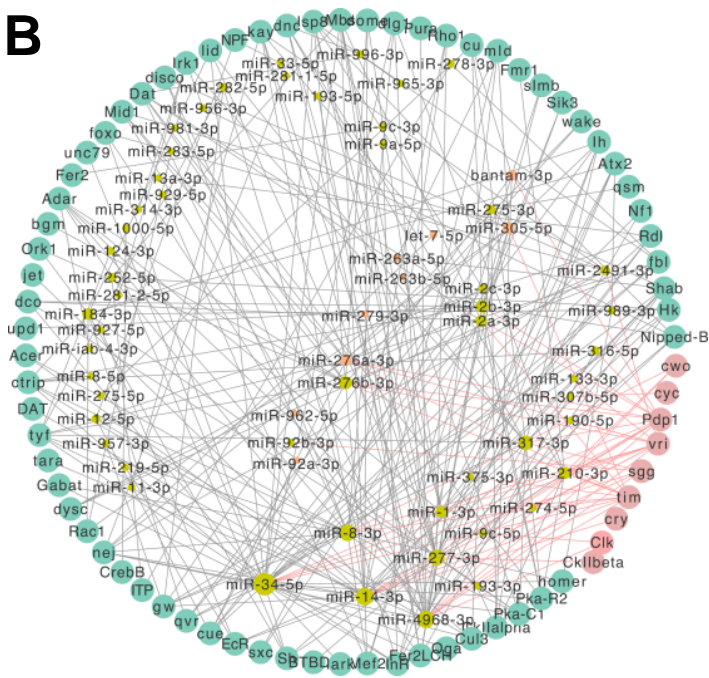

GO: biological process

chemical synaptic transmission

neurotransmitter secretion

glucose metabolic process dorsal closure

negative regulation of translation

cytoplasmic translation

nervous system development

wing morphogenesis

compound eye development sperm individualization
locomotor rhythm

asymmetric neuroblast division asymmetric neuroblast division deter-mation of adult lifespan inter-male aggressive behavio cell autophagic cell death
synaptic vesicle exocytosis

$$
\begin{array}{r}
\text { wing morphogenesis } \\
\text { axon guidance } \\
\text { sarcomere organization } \\
\text { sleep }
\end{array}
$$
visual perception regulation of cell shape cell adhesion border follicle cell migration myofibril assembly glucose metabolic process septate junction assembly actin filament organization phototransduction cell morphogenesis

cytoplasmic translation locomotor rhythm chemical synaptic transmission dorsal closure

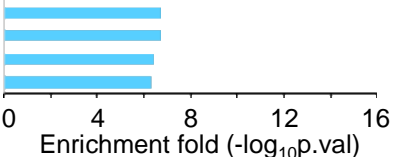

D

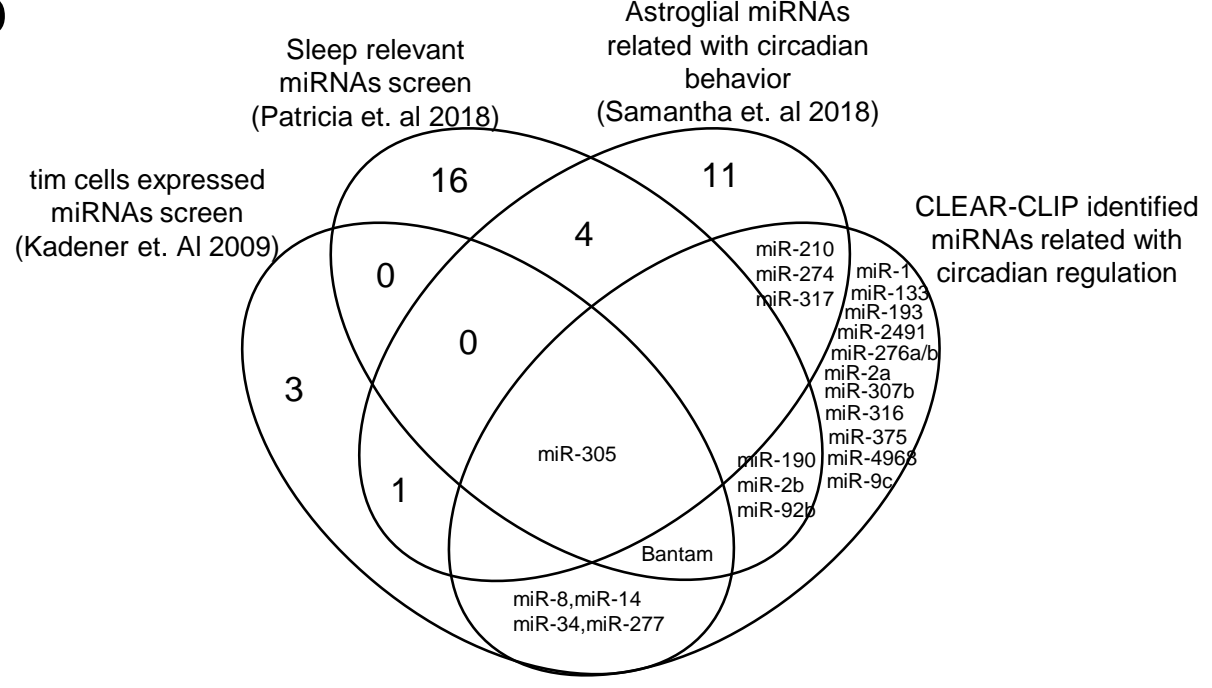


Fig. S3 The miRNA-mRNA interactions involved in Drosophila circadian system.

(A) Globally distinct miRNA-mRNA interactome of wild type $\mathrm{W}^{1118}$ and Clk $\mathrm{k}^{\mathrm{jrk}}$ mutant in whole body or head displayed as self-organized maps with the GEDIv2.1. Color bar indicates the normalized peak height.

(B) The miRNAs:circadian-genes interacting networks defined chimeras from Drosphila CLEAR-CLIP. The circadian genes were extracted from Flybase with phenotype emphasized in circadian defective behavior. These genes are aligned in circle, color in light red represents core genes in circadian system, color in light green stands for other circadian related genes. miRNAs in red have published results supporting circadian related functions or expression.

(C) GO enrichment analysis of chimeras-defined miRNA targets. The top twenty biological process terms were plotted.

(D) The Venn diagram of circadian-relevant miRNAs screen.

A

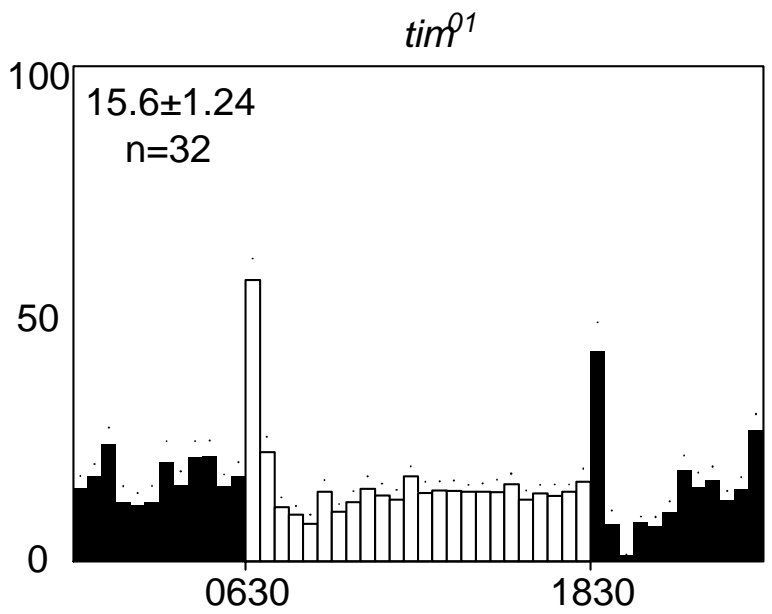

B

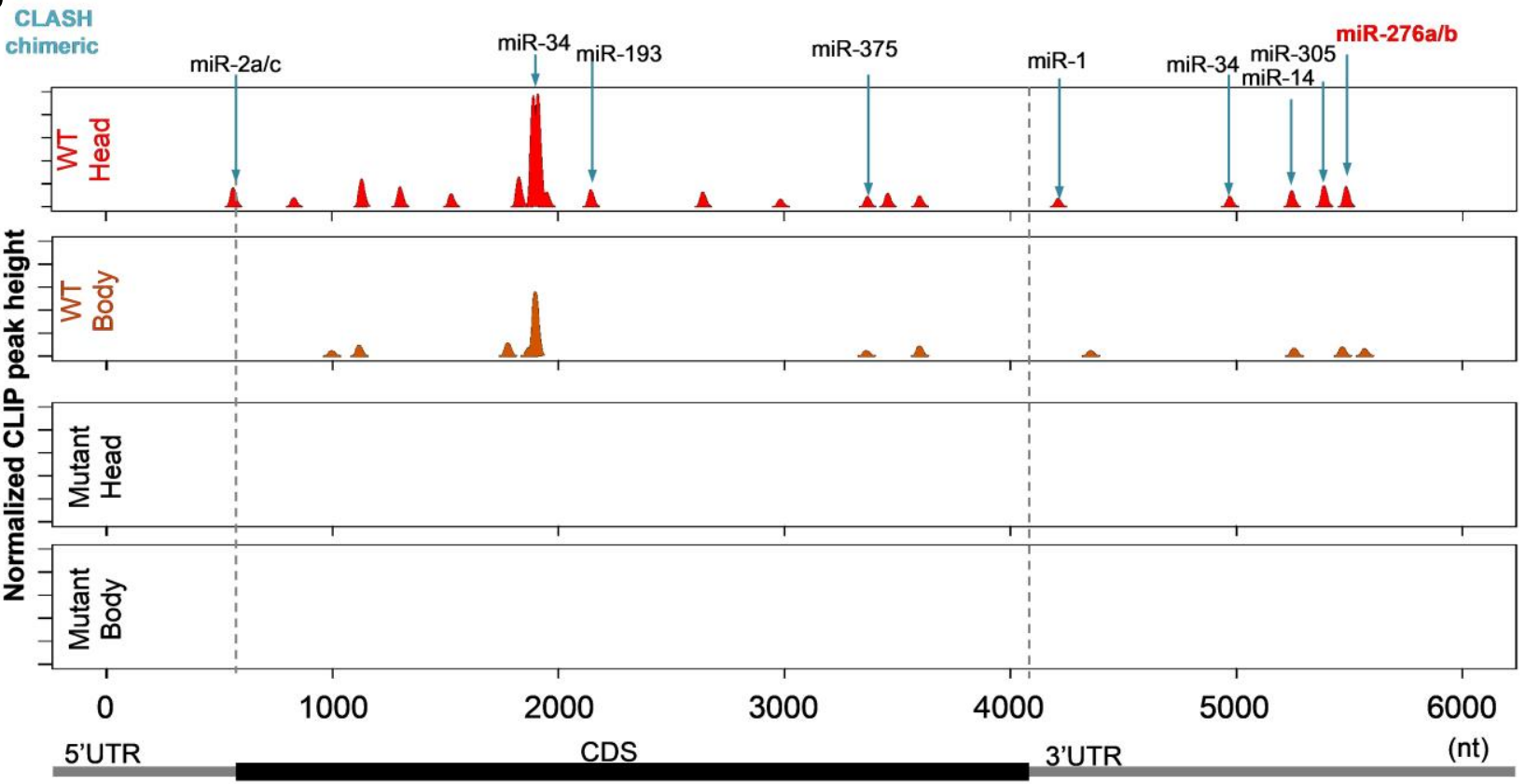

Flybase timeless transcript (FBtr0333253)

Fig. S4 The miRNAs involved regulation of tim in Drosophila.

(A) Locomotor profiles of tim01 in LD condition.

(B) The distribution of Ago1 peaks along the timeless transcript. miR-276a has been confirmed to target timelss. 
Table S1A. Read statistics for 8 drosophila CLEAR-CLIP samples.

\begin{tabular}{lllllll}
\hline & & & & Mapped & \multicolumn{2}{l}{ Chimeric } \\
sampleName Raw Reads & clean Reads & Uniq Reads & Reads & Reads & Percentage \\
\hline W01 & 22252916 & 22166118 & 2607404 & 1104091 & 18398 & $1.67 \%$ \\
W02 & 18229939 & 18194988 & 2204986 & 950071 & 16954 & $1.05 \%$ \\
W03 & 20063980 & 20005202 & 3417700 & 1578808 & 25779 & $1.02 \%$ \\
W04 & 48304093 & 47895896 & 4951811 & 2946369 & 68635 & $1.79 \%$ \\
C01 & 23493584 & 23438634 & 2356272 & 873423 & 17674 & $1.02 \%$ \\
C02 & 24206211 & 24047074 & 3333769 & 1296768 & 25325 & $1.03 \%$ \\
C03 & 11505949 & 11487289 & 1244821 & 464129 & 9733 & $1.05 \%$ \\
C04 & 45347628 & 45216440 & 4062840 & 2094932 & 51894 & $1.66 \%$ \\
\hline
\end{tabular}

Table S1B. Experimentally Validated miRNA Targets Found in chimeras supported interactions

\begin{tabular}{|c|c|c|c|}
\hline miRNA & Genes & Number of chimeric reads & Found in Experiments \\
\hline dme-bantam-3p & hid & 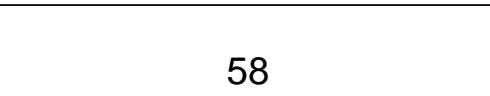 & $\begin{array}{l}\text { W1118(whole body), } \\
\text { W }^{1118} \text { (head) }\end{array}$ \\
\hline dme-let-7-5p & $a b$ & 12 & $W^{1118(h e a d)}$ \\
\hline dme-miR-11-3p & $\mathrm{E}(\mathrm{spl})$ malpha-BFM & 13 & $W^{1118}$ (whole body) \\
\hline dme-miR-124-3p & gli & 20 & clkjrk(head) \\
\hline dme-miR-1-3p & tutl & 14 & $W^{1118}$ (head) \\
\hline dme-miR-1-3p & CG18542 & 5 & $W^{1118}$ (whole body) \\
\hline dme-miR-1-3p & DI & 10 & clkjrk(Whole body),clkjik(head) \\
\hline dme-miR-1-3p & Msr-110 & 8 & clkjrk(whole body) \\
\hline dme-miR-1-3p & Chd64 & 13 & $W^{1118(h e a d)}$ \\
\hline dme-miR-14-3p & EcR & 5 & $W^{1118(h e a d)}$ \\
\hline dme-miR-14-3p & sug & 6 & $W^{1118}$ (whole body) \\
\hline dme-miR-14-3p & IP3K2 & 4 & $W^{1118}$ (whole body) \\
\hline dme-miR-34-5p & $\mathrm{Su}(\mathrm{z}) 12$ & 2 & $W^{1118}$ (whole body) \\
\hline dme-miR-7-5p & $\mathrm{E}(\mathrm{spl}) \mathrm{m} 3-\mathrm{HLH}$ & 1 & $W^{1118}$ (whole body) \\
\hline dme-miR-7-5p & $\mathrm{h}$ & 21 & $W^{1118}$ (head) \\
\hline dme-miR-8-3p & wls & 2 & clkjrk(whole body) \\
\hline dme-miR-8-3p & pan & 7 & clkjrk(whole body) \\
\hline
\end{tabular}

Table S1C. miRNA-first and miRNA-last distribution found in CLEAR-CLIP

\begin{tabular}{|c|c|c|c|c|}
\hline sample & \multicolumn{2}{|c|}{ W $^{1118}$} & \multicolumn{2}{c|}{ clkjk } \\
\hline Chimeric Reads & head & Whole body & Head & Whole body \\
\hline miRfirst chimeric & 4500 & 6697 & 2271 & 4151 \\
\hline miRlast chimeric & 269 & 590 & 214 & 346 \\
\hline
\end{tabular}


Table S2. The sequences of primer for real time PCR

\begin{tabular}{cc}
\hline tim- $y g-F$ & AGTCGCCACTCACCATTCCT \\
tim- $y g-R$ & CGTTGTTCTTCTTGCTCTGC \\
actin- $F$ & CCAACCGTGAGAAGATGA \\
actin- $R$ & GGAGTCCAGAACGATACC \\
$2 s$ & TGCTTGGACTACATATGGTTGAGGG \\
miR-375 & CGACGTTTGTTCGTTTGGCTTAAGTTA \\
miR- $9 c$ & GCGATTGTACTTCATCAGGTGCTCTG \\
miR-305 & GGCCGTCTTTGGTATTCTAGCTGTAGA \\
\hline
\end{tabular}

